\title{
A Constructive Proof of the Existence of Collateral Equilibrium for a Two-period Exchange Economy Based on a Smooth Interior-point Path
}

\author{
Wei Ma*
}

\begin{abstract}
In the real economic world, we often suffer from the default risk-the present financial crisis could serve as an evidence. The long established idea for inhibiting the default behavior is to resort to collateral, and this idea has profound effect on the market equilibrium. To study this effect, we make an investigation of the economy wherein default risk is present, assets are collateralized and households are risk-averse. We shall prove that the economy is in possession of an equilibrium. This proof is distinct from the existing one, in two respects: First it shows that equilibrium exists for the Leontief utility function, and second it is constructive. By constructive we mean that, on the basis of this proof, we can (and we shall) develop an algorithm for computing that equilibrium. The algorithm developed is of a path-following type. It starts from an economy which consists of several sub-economies, each with a complete market and a Cobb-Douglas utility function; and it terminates at the equilibrium of the economy under consideration. The algorithm is shown by simulation to be effective.
\end{abstract}

Keywords: Collateral; default; general equilibrium; incomplete market; path-following algorithm.

${ }^{*}$ Department of Economics, University of Pretoria, Pretoria 0002, South Africa. 


\section{Introduction}

It is well known that the now pervading global financial crisis stems from the U.S. sub-prime mortgage crisis. The sub-prime mortgage market is a kind of collateralized asset market, which features, alongside the standard asset market in the classical Arrow-Debreu model, the collateralization of assets and the presence of default risk. The collateralized asset market bulks large in the financial market; in 2009, the ratio of residential mortgages to GDP is 72.4 percent in U.S., 85.6 percent in U.K., and even in China, a now rising nation, more than 13 percent. These value statistics, in combination with the troubling financial crisis, indicate that this market commands a serious investigation.

It has been shown in Geanakoplos and Zame (2010) that the reliance on collateral to secure loans has substantial effect on the market equilibrium. In that literature, the authors made an extension of the intertemporal general equilibrium model by allowing for collateralized assets, default risk, and durable commodities, which can, aside from being consumed, serve as collateral. They proved the existence of equilibrium (called collateral equilibrium hereafter, or CE for short) for such an extended model, and conducted an analysis of its efficiency. Their proof is, however, an existence, instead of a constructive, one. The main object of this paper is therefore to seek a constructive proof of the existence of collateral equilibrium, so that a procedure for the computation thereof can be derived from it.

The economic phenomenon or behavior of default has received considerable attention in recent years. In order to study quantitatively who in an economy may benefit, and who may suffer, from the government regulation of collateral requirements, Araujo et al. (2012) specialize the Geanakoplos-Zame model to a case where all assets are endowed with a single and riskless promise, but each with distinct collateral requirements. They present there necessary and sufficient conditions for the collateral equilibrium to be Pareto-efficient by characterizing a minimal set of assets that may be traded in equilibrium. To investigate the effect of collateral requirements on dynamic behavior of economic agents, Gottardi and Kubler (2012) extend the Geanakoplos-Zame model to an infinite-horizon setting and have a discussion of the efficiency of competitive equilibria and the existence of Markov equilibria. ${ }^{1}$

In contrast to the articles quoted above, the focus of the present paper is on

\footnotetext{
${ }^{1}$ For other models on the infinite-horizon economy, one is referred to Araujo et al. (2002); Magill and Quinzii (1994). For other models involving default risk, see, for example, Diamond (1984); Dubey et al. (2005); Gale and Hellwig (1985); Sabarwal (2003); Townsend (1979); Zame (1993).
} 
the computation of collateral equilibria. The model we shall use is essentially the same as that of Geanakoplos and Zame (2010), except that the assumptions on the individual preference are slightly different. In Geanakoplos and Zame (2010), the utility function is assumed to be strictly monotone in date-zero consumption of perishable commodities, and in date-one consumption of all commodities. This assumption excludes, for example, the Leontief utility function. For this reason, we shall weaken it in this paper, by assuming the utility function to be weakly monotone in all commodities, both date-zero and date-one.

Under this assumption, we shall attempt to prove in Section 4 the existence of collateral equilibrium and find in Section 5 a procedure for its computation. The computation of collateral equilibrium is important for the purpose of comparative statics, and comparative-static properties constitute, as emphasized by Paul Samuelson, the main empirical implication of a model. In Dubey et al. (2005), punishment was invoked to inhibit the default behavior, and it was shown there that this way would not lower, and sometimes might promote, efficiency of the market equilibrium. By contrast, the efficiency of collateral equilibrium is affected greatly by the (exogenously given) collateral requirement level, whose optimal value should neither be too high nor too low. (See Section 3.) No doubt, an algorithm for computing collateral equilibrium will facilitate the determination of this optimal value.

As we know, algorithms of path-following type have long been used in economic equilibrium computation. Historically, it is Scarf (1967) who first introduced an algorithm of this type that makes possible the computation of economic equilibrium. Then Eaves developed in 1972 the Homotopy method (Eaves, 1972), and Smale in 1976 developed the global Newton method (Kellogg et al., 1976; Smale, 1976). For a review of these methods, we refer the reader to, among others, Allgower and Georg (1993); Eaves and Schmedders (1999); Scarf and Hansen (1973). In Brown et al. (1996), the authors devised a path-following algorithm for computing equilibria of the general equilibrium model with incomplete market. ${ }^{2}$ To compute collateral equilibrium, we shall in this paper try to develop an algorithm of this type. We shall show existence of such a path which starts from an economy where all households share a common generalized Cobb-Douglas utility function, no commodity is durable, and assets are all with zero collateral re-

\footnotetext{
${ }^{2}$ When the market is incomplete, Radner (1972) established the existence of Radner equilibrium on condition that the volume of trade in any asset is bounded above. Without this boundary condition equilibrium may fail to exist (Hart, 1975; Duffie and Shafer, 1985). Even it does, however, Pareto efficiency has become an ideal that is seldom attainable (See Diamond, 1967; Stiglitz, 1982). Plainly, collateral requirements would curtail one's borrowing capacity and creates a natural bound on the sales of assets, so existence of collateral equilibrium is guaranteed without imposing explicitly that boundary condition.
} 
quirement level; and terminates at the collateral equilibrium of the economy under consideration. The starting economy of the path is chosen in a way such that its equilibrium is unique and easy to find. It will be demonstrated in Section 6 that this algorithm is effective in terms of the accuracy of the equilibrium it provides.

\section{The Economy}

We study a pure exchange economy $\mathscr{E}$ with two dates (date 0 and date 1 ), $\hbar$ households, and $J$ assets. Let there be $S$ possible states of nature at date 1 and suppose the households own subjective probabilities as to their occurrence. Call date 0 state 0 . In each state, assume there are $L$ commodities available for trade and commodity $\ell$ in state $s$ will be indexed by $(s, \ell)$. The assets are traded at date 0 and pay off at date 1. Contrary to the classical economy, a seller of an asset in this economy is allowed to default on his promise, so is, in order for there to be a buyer, obliged to put up a prescribed amount of collateral to back his promise. The collateral should obviously be durable commodities. Assume it is held, and therefore can be consumed, by the borrower, and will be forfeited if he does default.

\subsection{Notation and assumption}

Given any two vectors $y^{1}=\left(y_{1}^{1}, \cdots, y_{n}^{1}\right), y^{2}=\left(y_{1}^{2}, \cdots, y_{n}^{2}\right)$ in the Euclidean space $\mathscr{R}^{n}$, let $y^{1} \geq y^{2}$ be that $y_{i}^{1} \geq y_{i}^{2}$ for all $i=1, \cdots, n ; y^{1}>y^{2}$ be that $y^{1} \geq y^{2}$ and $y^{1} \neq y^{2} ; y^{1} \gg y^{2}$ be that $y_{i}^{1}>y_{i}^{2}$ for all $i=1, \cdots, n ;\left\|y^{1}\right\|=\max _{i}\left|y_{i}^{1}\right|$, and $\left\|y^{1}\right\|_{1}=$ $\sum_{i}\left|y_{i}^{1}\right|$. For a scalar $b, y^{1} \geq b$ means $y_{i}^{1} \geq b$ for all $i=1, \cdots, n$, and, similarly, for $\geq$ and $\gg$. Suppose the consumption space $X$ of each household is $\mathscr{R}_{+}^{(S+1) L}$. Let $x^{h}=\left(x_{0}^{h}, x_{1}^{h}, \cdots, x_{S}^{h}\right)$ be a consumption bundle of household $h$, where $x_{s}^{h}$ is what he consumes in state $s ; e_{s}^{h}=\left(e_{s 1}^{h}, \cdots, e_{s L}^{h}\right)$ be his endowment in state $s$; and $u^{h}(x)$ : $X \rightarrow R$ be his utility function.

Given a consumption bundle $x_{0}^{h}$ at date 0 , let $F_{s}\left(x_{0}^{h}\right) \in \mathscr{R}_{+}^{L}$ be what remains of $x_{0}^{h}$ in state $s$ at date 1 . Suppose it is a positive linear transformation of $x_{0}^{h}$, that is, $F_{s}\left(x_{0}^{h}\right)=\kappa_{s} \circ x_{0}^{h}$, where $\kappa_{s} \in \mathscr{R}_{+}^{L}$ and the operator ' $\circ$ ' means Hadamard product. Let $\mathbf{S}=\{1, \cdots, S\}, \overline{\mathbf{S}}=\{0\} \cup \mathbf{S}, \mathbf{H}=\{1, \cdots, \hbar\}, \mathbf{L}=\{1, \cdots, L\} ; \mathbf{J}=\{1, \cdots, J\}$; let

$$
e_{0}=\sum_{h \in \mathbf{H}} e_{0}^{h}, \quad e_{s}=\sum_{h \in \mathbf{H}} e_{s}^{h}+F_{s}\left(e_{0}\right), \forall s \in \mathbf{S},
$$

and $e=\left(e_{0}, \cdots, e_{S}\right)$.

We make the following assumptions: 
(i) $e_{s}^{h} \gg 0$ for all $s \in \overline{\mathbf{S}}$ and $h \in \mathbf{H}$;

(ii) $u^{h}(x)$ is continuous, concave and weakly monotone in $x_{s l}, \forall(s, l) \in \overline{\mathbf{S}} \times \mathbf{L}$;

The first assumption requires each household to have a positive endowment of all commodities in all states, which is stronger than its counterpart in Geanakoplos and Zame (2010). For the assumption on utility function $u^{h}(x)$, it is assumed in the latter to be quasi-concave, strictly monotone in date-one consumption of all commodities and in date-zero consumption of all perishable commodities. This rules out the Leontief utility function. In contrast, $u^{h}(x)$ is assumed here to be weakly monotone in consumption of all commodities in all states. But for computational purpose, it is required to be concave.

\subsection{Real assets}

Real asset $j$ has its payoff $A^{j} \in \mathscr{R}_{+}^{S L}$ and is collateralized by a bundle $c^{j} \in \mathscr{R}_{+}^{L}$ of date-zero commodities. ${ }^{3}$ Let $A^{j}=\left(A_{1}^{j}, \cdots, A_{S}^{j}\right)$, where $A_{s}^{j} \in \mathscr{R}_{+}^{L}$ is the payoff of asset $j$ in state $s$. Since default is permitted, the actual delivery $\mathrm{DEL}_{s}^{j}$ of asset $j$ in state $s$ is given by

$$
\operatorname{DEL}_{s}^{j}=\min \left\{p_{s} \cdot A_{s}^{j}, p_{s} \cdot F_{s}\left(c^{j}\right)\right\},
$$

where $p_{s} \in \mathscr{R}_{+}^{L}$ is the spot price vector in state $s$.

\subsection{Households}

Household $h$ is assumed to be a price-taker and characterized by his utility function $u^{h}(x)$ and endowment $e^{h}$. For notational simplicity, we distinguish his asset purchases $\varphi^{h}$ and asset sales $\psi^{h}$, both in $\mathscr{R}_{+}^{J}$. Let $p=\left(p_{0}, \cdots, p_{S}\right) \in \mathscr{R}_{+}^{(S+1) L}$, where $p_{s}$ is the spot price vector of commodities in state $s$, and $q=\left(q_{1}, \cdots, q_{J}\right) \in \mathscr{R}_{+}^{J}$, where $q_{j}$ is the price of asset $j$.

Let $\eta^{h}=\left(x^{h}, \varphi^{h}, \psi^{h}\right)$ be a plan. The budget set $B^{h}(p, q)$ consists of such plans

\footnotetext{
${ }^{3}$ Here, only real assets is assumed to be available. However, the algorithm given below also works well when assets that are not real appear, if their payoffs are, or can be approximated by, smooth functions of the price vector. For instance, the payoff of a call or a put option can be approximated by the smooth function given in the next paragraph.
} 
that

$$
\begin{aligned}
& p_{0}\left(x_{0}^{h}-e_{0}^{h}\right)+q\left(\varphi^{h}-\psi^{h}\right) \leq 0 \\
& \sum_{j} \psi_{j}^{h} c^{j}-x_{0}^{h} \leq 0 \\
& \forall s \in \mathbf{S}, p_{s}\left(x_{s}^{h}-e_{s}^{h}\right)-p_{s} F_{s}\left(x_{0}^{h}\right)+\sum_{j}\left(\psi_{j}^{h}-\varphi_{j}^{h}\right) \mathrm{DEL}_{s}^{j} \leq 0
\end{aligned}
$$

where (2.2) implies that collateral is consumed by the borrower. Household $h$ then solves

$$
\left\{\max u^{h}\left(x^{h}\right):\left(x^{h}, \varphi^{h}, \psi^{h}\right) \in B^{h}(p, q)\right\}
$$

for his consumption bundle $x^{h}$, purchase portfolio $\varphi^{h}$ and sales portfolio $\psi^{h}$.

\subsection{Collateral equilibrium}

DEFINITION 1. A collateral equilibrium is a collection $\left\{p, q,\left(\eta^{h}\right)_{h \in \mathbf{H}}\right\}$ such that

- Utility maximization: Given $(p, q), \eta^{h}$ solves (2.4), for all $h \in \mathbf{H}$;

- Commodity markets at date 0 clear: $\sum_{h \in \mathbf{H}}\left(x_{0}^{h}-e_{0}^{h}\right)=0$;

- Commodity markets at date 1 clear: $\sum_{h \in \mathbf{H}}\left(x_{s}^{h}-e_{s}^{h}-F_{s}\left(x_{0}^{h}\right)\right)=0$, for all $s \in \mathbf{S}$;

- Asset markets clear: $\sum_{h \in \mathbf{H}}\left(\varphi^{h}-\psi^{h}\right)=0$.

\subsection{GEI equilibrium}

Assume the number $J$ of assets is less than the number $S$ of states at date 1 . And assume that the promise of each asset is kept and no collateral is required. Replace $\mathrm{DEL}_{s}^{j}$ in (2.3) with $A_{s}^{j}$, and we get

$$
p_{s}\left(x_{s}^{h}-e_{s}^{h}\right)-p_{s} F_{s}\left(x_{0}^{h}\right)+\sum_{j}\left(\psi_{j}^{h}-\varphi_{j}^{h}\right) A_{s}^{j} \leq 0 .
$$

The budget set $\bar{B}^{h}(p, q)$ of household $h$ is now defined to be the set of plans that satisfy $(2.1)$ and $\left(2.3^{\prime}\right)$, and he solves

$$
\left\{\max u^{h}\left(x^{h}\right): \eta^{h} \in \bar{B}^{h}(p, q)\right\}
$$

for his plan $\eta^{h}$.

DEFINITION 2. A GEI equilibrium is a collection $\left\{p, q,\left(\eta^{h}\right)_{h \in \mathbf{H}}\right\}$ such that

- Utility maximization: Given $(p, q), \eta^{h}$ solves $\left(2.4^{\prime}\right)$, for all $h \in \mathbf{H}$; 
- Commodity markets at date 0 clear: $\sum_{h \in \mathbf{H}}\left(x_{0}^{h}-e_{0}^{h}\right)=0$;

- Commodity markets at date 1 clear: $\sum_{h \in \mathbf{H}}\left(x_{s}^{h}-e_{s}^{h}-F_{s}\left(x_{0}^{h}\right)\right)=0$, for all $s \in \mathbf{S}$;

- Asset markets clear: $\sum_{h \in \mathbf{H}}\left(\varphi^{h}-\psi^{h}\right)=0$.

Note that the market clearing conditions are the same as those in Definition 1.

\section{An Example}

The example is adapted from Example 2 of Geanakoplos and Zame (2010). The adaption is: Households are here free to own subjective probability distributions, rather than sharing an objective one, as to the occurrence of date-one states. This example is presented with a dual purpose: first, to illustrate how collateral requirements affect the market equilibria and the social welfare; second, to show that distinct beliefs on the occurrence of date-one states would influence the optimal collateral requirement and the social welfare.

More specifically, it will be shown later (i) that different collateral requirements would lead to different equilibria with different levels of social welfare; and (ii) that when both households in the economy agree on the probabilities of default, the optimal collateral requirement level is given by $1 / 4$, in which case household 2 will default on his promise in equilibrium, but when they do not agree on the probabilities of default, the optimal collateral requirement level is given, instead, by $1 / 3$, and household 2 will choose not to default in equilibrium, even though he is entitled to do so.

EXAMPLE 3.1. Suppose there are two states of nature at date 1, and two commodities are available for trade in each state: Food, which is perishable, and Housing, which is perfectly durable. There are two types of households in the economy. The utility function and endowment for the first type is given by

$$
\begin{aligned}
& u^{1}=x_{0 F}^{1}+x_{0 H}^{1}+\gamma_{11}\left(x_{1 F}^{1}+x_{1 H}^{1}\right)+\gamma_{12}\left(x_{2 F}^{1}+3 x_{2 H}^{1}\right) \\
& e^{1}=(29 / 2,1 ; 9,0 ; 9,0) ;
\end{aligned}
$$

and, for the second type, by

$$
\begin{aligned}
u^{2} & =\log x_{0 F}^{2}+4 x_{0 H}^{2}+\gamma_{21}\left(x_{1 F}^{2}+4 x_{1 H}^{2}\right)+\gamma_{22}\left(x_{2 F}^{2}+4 x_{2 H}^{2}\right) \\
e^{1} & =(7 / 2,0 ; 9,0 ; 5 / 2,0) ;
\end{aligned}
$$

where $\gamma_{i j}, i, j=1,2$ are nonnegative scalars satisfying $\gamma_{11}+\gamma_{12}=\gamma_{21}+\gamma_{22}=1$. 
Suppose a single security promising one unit of food at date 1 and collateralized by $\lambda$ unit of housing is available for trade. The equilibrium of the economy depends on the value of $\lambda$ and is given below. For detailed computation, please refer to Geanakoplos and Zame (2010). Let $x^{i}=\left(x_{0 F}^{i}, x_{0 H}^{i} ; x_{1 F}^{i}, x_{1 H}^{i} ; x_{2 F}^{i}, x_{2 H}^{i}\right), i=1,2, p=$ $\left(p_{0 F}, p_{0 H} ; p_{1 F}, p_{1 H} ; p_{2 F}, p_{2 H}\right)$, and the social welfare $W$ be $\left(u^{1}+u^{2}\right)$.

(i) $\lambda=0$

Since the security is not backed by any collateral, its delivery will be zero, and so will its price. Household 1 is not willing to buy the security and household 2 can borrow nothing. The equilibrium is given by

$$
\begin{aligned}
q_{0} & =0, \\
p & =(1,5 ; 1,4 ; 1,4), \\
x^{1} & =\left(18-\frac{5}{8}, \frac{17}{40} ; \frac{107}{10}, 0 ; \frac{107}{10}, 0\right), \\
x^{2} & =\left(\frac{5}{8}, \frac{23}{40} ; \frac{73}{10}, 1 ; \frac{4}{5}, 1\right) .
\end{aligned}
$$

(ii) $\lambda \in(0,1 / 4)$

If household 2 borrows nothing, his marginal utility of income at date 0 is $8 / 5$; otherwise, it is

$$
\frac{8-\left(4 \gamma_{21}+3 \gamma_{22}\right)}{5-\left(4 \gamma_{11}+3 \gamma_{12}\right)} \geq \frac{4}{2}>\frac{8}{5} ;
$$

so he will borrow as much as possible, or $4 \gamma_{11}+3 \gamma_{12}$, which is independent of the collateral level. Note competition will drive $p_{0 H}$ above 5. The equilibrium is given by

$$
\begin{aligned}
q_{\lambda} & =\left(4 \gamma_{11}+3 \gamma_{12}\right) \lambda, \\
p & =\left(1,4 \gamma_{11}+3 \gamma_{12}+\frac{7}{2} \cdot \frac{8-4 \gamma_{21}-3 \gamma_{22}}{9-4 \gamma_{21}-3 \gamma_{22}} ; 1,4 ; 1,3\right), \\
x^{1} & =\left(18-\frac{7}{2\left(9-4 \gamma_{21}-3 \gamma_{22}\right)}, 0 ; 13,0 ; \frac{23}{2}, \frac{1}{6}\right), \\
x^{2} & =\left(\frac{7}{2\left(9-4 \gamma_{21}-3 \gamma_{22}\right)}, 1 ; 5,1 ; 0, \frac{5}{6}\right) .
\end{aligned}
$$

(iii) $\lambda \in[1 / 4,1 / 3)$

As above, household 2 will borrow up to his limit $\gamma_{11} / \lambda+3 \gamma_{12}$, which varies 
inversely with the collateral requirement level. The equilibrium is given by

$$
\begin{aligned}
q_{\lambda} & =\gamma_{11}+3 \lambda \gamma_{12}, \\
p & =\left(1, \frac{q_{\lambda}}{\lambda}+\frac{7}{2} \cdot \frac{8-\gamma_{21} / \lambda-3 \gamma_{22}}{9-\gamma_{21} / \lambda-3 \gamma_{22}} ; 1,4 ; 1,3\right), \\
x^{1} & =\left(18-\frac{7}{2\left(9-\gamma_{21} / \lambda-3 \gamma_{22}\right)}, 0 ; 9+\frac{1}{\lambda}, 0 ; \frac{23}{2}, \frac{1}{6}\right), \\
x^{2} & =\left(\frac{7}{2\left(9-\gamma_{21} / \lambda-3 \gamma_{22}\right)}, 1 ; 9-\frac{1}{\lambda}, 1 ; 0, \frac{5}{6}\right) .
\end{aligned}
$$

Marginal utility of income $M U_{0}^{1}$ of household 1 at date 0 is invariably unity, so his utility is maximized when $p_{0 H}$ peaks; that of household 2 at date 0 is

$$
M U_{0}^{2}=\frac{2\left(9-\gamma_{21} / \lambda-3 \gamma_{22}\right)}{7}
$$

which varies directly as $\lambda$. Differentiating $p_{0 H}$ with respect to $\lambda$, we obtain

$$
\frac{d p_{0 H}}{d \lambda}=-\frac{1}{\lambda^{2}}\left[\gamma_{11}-\frac{7}{2} \cdot \frac{\gamma_{21}}{\left(9-\gamma_{21} / \lambda-3 \gamma_{22}\right)^{2}}\right]
$$

and differentiating the social welfare $W$ with respect to $\lambda$, we obtain

$$
\frac{d W}{d \lambda}=-\frac{1}{\lambda^{2}}\left[\left(\gamma_{11}-\frac{7}{2} \cdot \frac{\gamma_{21}}{\left(9-\gamma_{21} / \lambda-3 \gamma_{22}\right)^{2}}\right)-\gamma_{21}\left(1-\frac{1}{9-\gamma_{21} / \lambda-3 \gamma_{22}}\right)\right] .
$$

When $\gamma_{11}=\gamma_{21}, d W / d \lambda$ is inside zero; so at $\lambda=1 / 4$ social welfare has its peak and satisfaction of household 2 meets its trough, and he will prefer to default in state 2 . But when $\gamma_{11}$ is substantially less than $\gamma_{21}, d W / d \lambda$ is beyond zero; both social welfare and satisfaction of household 2 have their peaks at $\lambda=1 / 3$; and he will, at this level, be ready to stand by his promise at date 1 .

Suppose the objective probability distribution is given by $(0.95,0.05)$ and both households enjoy it, then social welfare is maximized at $\lambda=1 / 4$ and the corresponding value stands at 42.58. If household 1 takes a quite different view $\left(\gamma_{11}, \gamma_{12}\right)=(0.05,0.95)$, social welfare then achieves its maximum at $\lambda=1 / 3$ and its value falls to 42.54 .

(iv) $\lambda \in[1 / 3,2 / 5)$

Household 2 will again borrow up to his limit $1 / \lambda$, which is, contrary to the case above, independent of the subjective beliefs of the households. The 
equilibrium is given by

$$
\begin{aligned}
q_{\lambda} & =1, \\
p & =\left(1, \frac{1}{\lambda}+\frac{7}{2} \cdot \frac{8 \lambda-1}{9 \lambda-1} ; 1,4 ; 1,3\right), \\
x^{1} & =\left(18-\frac{7 \lambda}{2(9 \lambda-1)}, 0 ; 9+\frac{1}{\lambda}, 0 ; \frac{23}{2}, \frac{1}{3 \lambda}-\frac{5}{6}\right), \\
x^{2} & =\left(\frac{7 \lambda}{2(9 \lambda-1)}, 1 ; 9-\frac{1}{\lambda}, 1 ; 0, \frac{11}{6}-\frac{1}{3 \lambda}\right) .
\end{aligned}
$$

It is easily checked that

$$
\frac{d p_{0 H}}{d \lambda}<0, \quad \frac{d M U_{0}^{2}}{d \lambda}>0,
$$

and

$$
\frac{d W}{d \lambda}<0, \quad \text { when } \gamma_{22}<\frac{3 \lambda(11 \lambda-2)}{2(9 \lambda-1)} \text {. }
$$

Therefore, household 1 maximizes his utility at $\lambda=1 / 3$, and household 2 at $\lambda=2 / 5$. Social welfare will be maximized at $\lambda=1 / 3$, if household 2 owns the objective probability distribution.

(v) $\lambda \in[2 / 5,1 / 2)$

Household 2 can still borrow enough to buy all housing at date 0 ; but growing collateral level curtails his borrowing capacity, which permits him to deliver less at date 1 and enables him to consume some food in state 2. The equilibrium is given by

$$
\begin{aligned}
q_{\lambda} & =1, \\
p & =\left(1, \frac{1}{\lambda}+\frac{7}{2} \cdot \frac{8 \lambda-1}{9 \lambda-1} ; 1,4 ; 1,4\right), \\
x^{1} & =\left(18-\frac{7 \lambda}{2(9 \lambda-1)}, 0 ; 9+\frac{1}{\lambda}, 0 ; 9+\frac{1}{\lambda}, 0\right), \\
x^{2} & =\left(\frac{7 \lambda}{2(9 \lambda-1)}, 1 ; 9-\frac{1}{\lambda}, 1 ; \frac{5}{2}-\frac{1}{\lambda}, 1\right) .
\end{aligned}
$$

Similarly, we have

$$
\frac{d p_{0 H}}{d \lambda}<0, \quad \frac{d M U_{0}^{2}}{d \lambda}>0, \quad \frac{d W}{d \lambda}<0 .
$$

Therefore, household 1 maximizes his utility at $\lambda=2 / 5$, and household 2 at $\lambda=1 / 2$. Social welfare will be maximized at $\lambda=2 / 5$. 
(vi) $\lambda \in[1 / 2, \infty)$

At such high a collateral level, household 2 fails to raise enough money to finance his housing purchase at date 0 , so both households will share the one unit housing at that date. The equilibrium is given by

$$
\begin{aligned}
q_{\lambda} & =1, \\
p & =(1,5 ; 1,4 ; 1,4), \\
x^{1} & =\left(18-\frac{5 \lambda-1}{8 \lambda-1}, 1-\frac{7 \lambda}{2(5 \lambda-1)}+\frac{\lambda}{8 \lambda-1} ; 9+4 x_{0 H}^{1}+\frac{x_{0 H}^{2}}{\lambda}, 0 ; 9+\frac{1}{\lambda}, 0\right), \\
x^{2} & =\left(\frac{5 \lambda-1}{8 \lambda-1}, \frac{7 \lambda}{2(5 \lambda-1)}-\frac{\lambda}{8 \lambda-1} ; 5+\left(4-\frac{1}{\lambda}\right) x_{0 H}^{2}, 1 ; \frac{5}{2}-\frac{1}{\lambda}, 1\right) .
\end{aligned}
$$

For household 1, with the increased collateral level his sales of housing decreases, which, in turn, reduces his income at date 0 and lowers his utility level. For household 2, increased collateral level would diminish his marginal utility of income at date 0 , so both households favor a lower collateral requirement and social welfare is maximized at $\lambda=1 / 2$.

(vii) GEI equilibrium

Suppose only the security with payoff $\left(p_{1 F}, p_{2 F}\right)$ is available for trade. Household 2 is free to borrow without any collateral requirement. The GEI equilibrium is given by

$$
\begin{aligned}
q & =1 ; \varphi_{1}=5 ; \varphi_{2}=-5 ; \\
p & =(1,7.5 ; 1,4,1,3), \\
x^{1} & =\left(17,0 ; 14,0 ; \frac{23}{2}, \frac{5}{6}\right), \\
x^{2} & =\left(1,1 ; 4,1 ; 0, \frac{1}{6}\right) .
\end{aligned}
$$

It is easily checked that $p_{0 H}$ at this equilibrium is higher than that any collateral equilibrium, and so is the utility level of household 1. But that of household 2 slumps to a record low.

In sum, for CE, if both households believe the objective probability distribution, social welfare achieves its maximum 42.58 at $\lambda=1 / 4$; at this level, household 2 would default and the conclusion that default can promote efficiency holds. But if household 1, instead, believes $\left(\gamma_{11}, \gamma_{12}\right)=(0.05,0.95)$, then social welfare achieves its maximum 42.54 at $\lambda=1 / 3$, so a dead-weight loss results. As opposed to the case above, household 2 would now keep his promise and default fails to 
promote efficiency. Given the objective probability distribution, social welfare at the GEI equilibrium attains 42.63 , higher than that at every collateral equilibrium.

Recall that one of the insights of Geanakoplos and Zame (2010)(p. 4) is that

.. collateral requirements that lead to default (with positive probability) in equilibrium may (ex ante) Pareto dominate collateral requirements that do not lead to default. Moreover, if securities offering the same promise but backed by different collateral requirements are offered, the market may choose a collateral requirement that leads to default (with positive probability).

In the case of this example, it can be seen from the calculation above that this insight holds valid when the households agree on the probabilities of default; but otherwise it would cease to be true.

\section{Existence of Collateral Equilibrium}

Remember that the purpose of this paper is to compute the collateral equilibrium of an economy with the aid of a path-following algorithm. The strategy of a pathfollowing algorithm is to first deform the original economy to obtain a family of economies, one of which has a unique and easily accessible equilibrium; then prove that there exists a path departing from this unique equilibrium and terminating at the equilibrium of the original economy; and finally follow this path to secure the equilibrium of the original economy. Central to this algorithm is how to deform the original economy to obtain that desired family of economies. Let us treat this issue in what follows.

\subsection{An auxiliary economy}

For a vector $v=\left(v_{1}, \cdots, v_{n}\right)$, let $\ln v=\sum_{i=1}^{n} \ln v_{i}$; for a plan $\eta=(x, \varphi, \psi)$, let

$$
\phi(\eta ; \lambda)=\sum_{s \in \mathbf{S}} \ln x_{s}+\ln \varphi+\ln \psi+\ln \left(x_{0}-\lambda \sum_{j \in \mathbf{J}} \psi_{j} c^{j}\right) .
$$

From the standpoint of economics, the function $\phi$ is a (generalized) Cobb-Douglas utility function when $\lambda$ vanishes. It is to be noted that $\phi$ has as its arguments both the purchasing portfolio $\varphi$ and the sale portfolio $\psi$, so the question arises naturally whether they will go to infinity at equilibrium, if $\phi$ is adopted as a utility function. 
The answer is no; the budget constraints, as will be seen later, prevents them from blowing up at equilibrium. From the standpoint of optimization, the function $\phi$ serves as a barrier function, used to handle the boundary conditions.

For purely computational purpose, we add to the utility function $u^{h}$ a perturbation term, which vanishes as $\lambda$ is equal to unity, to get

$$
\tilde{u}^{h}(\eta ; \lambda)=u^{h}(x)+(1-\lambda) \sum_{i=1}^{N_{1}} \sigma_{i}^{h} \eta_{i}
$$

where $N_{1}=(S+1) L+2 J$ and $\sigma^{h}=\left(\sigma_{1}^{h}, \cdots, \sigma_{N_{1}}^{h}\right) \in \mathscr{R}_{+}^{N_{1}}$; and then we deform it by multiplying it with a factor $\lambda$, giving

$$
\bar{u}^{h}(\eta ; \lambda)=\lambda u^{h}(x)+\lambda(1-\lambda) \sum_{i=1}^{N_{1}} \sigma_{i}^{h} \eta_{i}
$$

finally we add the barrier function $\phi$, as a standard method to treat the boundary conditions in the theory of optimization, to this deformed utility function, to get

$$
U^{h}(\eta ; \lambda)=\bar{u}^{h}(\eta ; \lambda)+\lambda_{0}(1-\lambda) \phi(\eta ; \lambda),
$$

where $\lambda_{0}$ is a positive scalar. Of the value of $\lambda_{0}$ the existence of equilibrium is independent; but its choice will affect the performance of the algorithm developed later on.

It can easily be seen that $U^{h}$ is strictly monotone in consumption of all goods and in the purchasing portfolio $\varphi .{ }^{4}$ When $\lambda$ vanishes, it becomes $\phi(\eta ; 0)$, a CobbDouglas utility function, as stated above. We shall utilize $U^{h}$ as a utility function to define an auxiliary economy.

Again for computational purpose, we have to ensure that, when $\lambda$ vanishes, the equilibrium of the auxiliary economy is unique and easy to find. For this reason, let us deform the budget constraints, by defining $\tilde{B}^{h}(p, q, \lambda)$ to be a set of plans $(x, \varphi, \psi)$ that satisfies

$$
\begin{aligned}
& p_{0}\left(x_{0}^{h}-e_{0}^{h}\right)+\lambda q\left(\varphi^{h}-\psi^{h}\right)+(1-\lambda) q\left(\varphi^{h}+\psi^{h}-e_{a}^{h}\right) \leq 0 \\
& \forall s \in \mathbf{S}, p_{s}\left(x_{s}^{h}-e_{s}^{h}\right)-\lambda p_{s} F_{s}\left(x_{0}^{h}\right)+\lambda \sum_{j}\left(\psi_{j}^{h}-\varphi_{j}^{h}+(1-\lambda) e_{a j}^{h}\right) \mathrm{DEL}_{s}^{j} \leq 0
\end{aligned}
$$

\footnotetext{
${ }^{4}$ Its monotonicity in the sales portfolio $\psi$ is vague. Fortunately, this is not required in the present paper.
} 
where $e_{a}^{h}=\left(e_{a 1}^{h}, \cdots, e_{a J}^{h}\right) \in \mathscr{R}_{++}^{J}$, and wherein the collateral requirement of each asset and the durability of each commodity are all discounted by a factor of $\lambda$. Let $\tilde{\tilde{B}}^{h}(p, q, \lambda)$ be a subset of $\tilde{B}^{h}(p, q, \lambda)$ with each of its elements respecting

$$
\lambda \sum_{j} \psi_{j}^{h} c^{j}-x_{0}^{h} \leq 0
$$

which, at $\lambda=0$, reduces itself to the boundary condition $x_{0}^{h} \leq 0$.

When $\lambda$ vanishes, the budget set $\tilde{B}^{h}(p, q, \lambda)$ becomes

$$
\begin{aligned}
& p_{0}\left(x_{0}^{h}-e_{0}^{h}\right)+q\left(\varphi^{h}+\psi^{h}-e_{a}^{h}\right) \leq 0, \\
& \forall s \in \mathbf{S}, p_{s}\left(x_{s}^{h}-e_{s}^{h}\right) \leq 0 .
\end{aligned}
$$

From this set one can see that the assets have lost their ability to transfer wealth across states; instead they yield utility directly, just the same as a commodity. The vector $e_{a}^{h}$ can be seen as endowments of the assets, serving to prevent $\varphi^{h}$ and $\psi^{h}$ from blowing up when $\lambda$ is small.

We are now in a position to define the auxiliary economy $\mathscr{E}(\lambda)$, wherein household $h$ acts to optimize the utility function $U^{h}$, instead of $u^{h}$, with respect to $\tilde{B}^{h}(p, q, \lambda)$. It is to be noticed that $\mathscr{E}(1)=\mathscr{E}$, i.e., the original economy is recovered when $\lambda$ changes to one.

In the economy $\mathscr{E}(0)$, each household optimizes his behavior by solving

$$
\begin{aligned}
\max & \sum_{s \in \overline{\mathbf{S}}} \ln x_{s}+\ln \varphi+\ln \psi \\
& p_{0}\left(x_{0}^{h}-e_{0}^{h}\right)+q\left(\varphi^{h}+\psi^{h}-e_{a}^{h}\right) \leq 0, \\
& \forall s \in \mathbf{S}, p_{s}\left(x_{s}^{h}-e_{s}^{h}\right) \leq 0 .
\end{aligned}
$$

As the utility function is additive, this problem is equivalent to $(S+1)$ subproblems, each defined by, for $s=0$,

$$
\begin{array}{ll}
\max & \ln x_{0}+\ln \varphi+\ln \psi \\
& p_{0}\left(x_{0}^{h}-e_{0}^{h}\right)+q\left(\varphi^{h}+\psi^{h}-e_{a}^{h}\right) \leq 0 ;
\end{array}
$$

and for $s \in \mathbf{S}$,

$$
\max \ln x_{s} \quad \text { s.t. } p_{s}\left(x_{s}^{h}-e_{s}^{h}\right) \leq 0 .
$$

Each of these subproblems corresponds to an economy with a Cobb-Douglas utility function and a complete market, whose equilibrium is well known to be unique and readily accessible. The equilibrium of $\mathscr{E}(0)$ is, therefore, unique and readily accessible. We shall next show that the auxiliary economy $\mathscr{E}(\lambda)$ in general admits of an equilibrium. 


\subsection{The existence theorem}

DEFINITION 3. The equilibrium of $\mathscr{E}(\lambda)$ is a collection $\left\{p, q,\left(\eta^{h}\right)_{h \in \mathbf{H}}\right\}$ such that

- Given $(p, q), \eta^{h}$ optimizes $U^{h}$ in $\tilde{B}^{h}(p, q)$, for all $h \in \mathbf{H}$;

- Commodity markets at date 0 clear: $\sum_{h \in \mathbf{H}}\left(x_{0}^{h}-e_{0}^{h}\right)=0$;

- Commodity markets at date 1 clear: $\sum_{h \in \mathbf{H}}\left(x_{s}^{h}-e_{s}^{h}-\lambda F_{s}\left(x_{0}^{h}\right)\right)=0$, for all $s \in \mathbf{S}$

- Asset markets clear: $\sum_{h \in \mathbf{H}}\left[\lambda\left(\varphi^{h}-\psi^{h}\right)+(1-\lambda)\left(\varphi^{h}+\psi^{h}-e_{a}^{h}\right)\right]=0$.

We claim that

THEOREM 4.1. If assumptions (1), (2) are satisfied, and $c^{j}>0$ for all $j \in \mathbf{J}$, then $\mathscr{E}(\lambda)$ has an equilibrium for all $\lambda \in[0,1)$.

Proof. See the Appendix.

With the aid of this theorem, we can show that

THEOREM 4.2. Given assumptions in Theorem 4.1, the economy $\mathscr{E}$ has a collateral equilibrium.

ProOF. Let $\left\{\lambda^{k}\right\}$ be an increasing sequence of positive scalars such that $\lim _{k \rightarrow \infty} \lambda^{k}=$ 1; and let $\left(p^{k}, q^{k},\left(\eta_{k}^{h}\right)_{h \in \mathbf{H}}\right)$ be an equilibrium of $\mathscr{E}\left(\lambda^{k}\right)$. As shown in the proof of Theorem 4.1, the set $\left\{\left(p^{k}, q^{k},\left(\eta_{k}^{h}\right)_{h \in \mathbf{H}}\right): k=1,2, \cdots\right\}$ is uniformly bounded. Therefore, it has a convergent subsequence; without loss of generality, let it also be $\left\{\left(p^{k}, q^{k},\left(\eta_{k}^{h}\right)_{h \in \mathbf{H}}\right)\right\}_{k=1}^{\infty}$ and suppose it converges to $\left(p, q,\left(\eta^{h}\right)_{h \in \mathbf{H}}\right)$. We say that this is a collateral equilibrium of $\mathscr{E}$. Indeed, $\left(\eta^{h}\right)_{h \in \mathbf{H}}$ clear all markets, so it remains to show that

$$
\eta^{h} \in \underset{\eta}{\operatorname{argmax}}\left\{u^{h}(\eta): \eta \in B^{h}(p, q) \cap \square^{h}\right\} .
$$

By contradiction, suppose there exists an $\eta_{0} \in B^{h}(p, q) \cap \square^{h}$ such that $u^{h}\left(\eta_{0}\right)>$ $u^{h}\left(\eta^{h}\right)$. By the continuity of $u^{h}$, there is an $\alpha \in(0,1)$ satisfying $u^{h}\left(\alpha \eta_{0}\right)>u^{h}\left(\eta^{h}\right)$; so, for $k$ sufficiently large, $U^{h}\left(\alpha \eta_{0} ; \lambda^{k}\right)>U^{h}\left(\eta_{k}^{h} ; \lambda^{k}\right)$. Note that, by assumption (1), an $\alpha$ strictly less than one would render slack both constraints (2.1) and (2.3) at $\alpha \eta_{0}{ }^{5}$ Hence, when $k$ is sufficiently large, we have $\alpha \eta_{0} \in \tilde{B}^{h}\left(p^{k}, q^{k}, \lambda^{k}\right) \cap \square^{h}$. But this contradicts that household $h$ has optimized in the economy $\mathscr{E}\left(\lambda^{k}\right)$. Q.E.D

\footnotetext{
${ }^{5}$ In Dubey et al. (2005), this is called the scaling property of the budget set. This property also obtains, even if the budget set contains assets that are not real; in which case, it is easily verified that both Theorems 4.1 and 4.2 are still true.
} 


\section{Computation of Collateral Equilibrium}

For the price vectors $p_{s}$ such that $p_{s} \cdot A_{s}^{j}=p_{s} \cdot F_{s}\left(c^{j}\right), \mathrm{DEL}_{s}^{j}$ is non-differentiable. To facilitate the CE computation, we wish to approximate it by a smooth function $D_{s}^{j}(\lambda)$ given in Zang (1980), which is as follows:

$$
D_{s}^{j}(\lambda)= \begin{cases}p_{s} \cdot A_{s}^{j} & \text { if } \rho_{s}^{j} \leq-(1-\lambda), \\ -\frac{\left(\rho_{s}^{j}\right)^{2}}{4(1-\lambda)}+\frac{1}{2}\left(p_{s} \cdot A_{s}^{j}+p_{s} \cdot F_{s}\left(c^{j}\right)\right)-\frac{1}{4}(1-\lambda) & \text { if }-(1-\lambda)<\rho_{s}^{j} \leq(1-\lambda), \\ p_{s} \cdot F_{s}\left(c^{j}\right) & \text { if }(1-\lambda) \leq \rho_{s}^{j},\end{cases}
$$

where $\rho_{s}^{j}=p_{s} \cdot A_{s}^{j}-p_{s} \cdot F_{s}\left(c^{j}\right)$ and $\lambda \in[0,1]$. Furthermore, we assume the utility function $u^{h}(x)$ to be smooth.

\subsection{Characterization of the auxiliary economy}

As stated above, the equilibrium of $\mathscr{E}(0)$ exists and is unique. Denote it by $\left\{p(0), q(0),\left(x^{h}(0), \varphi^{h}(0), \psi^{h}(0)\right)\right.$ Construct a set of smooth and positive functions $\left\{\left(e^{h}(\lambda), e_{a}^{h}(\lambda)\right): h \in \mathbf{H}\right\}$ such that

$$
e^{h}(0)=x^{h}(0), e^{h}(1)=e^{h} ; \quad e_{a}^{h}(0)=\varphi^{h}(0)+\psi^{h}(0), e_{a}^{h}(1)=e_{a}^{h} .
$$

Household $h$ in $\mathscr{E}(\lambda)$ is then supposed to be endowed with a vector $e^{h}(\lambda)$ of commodities and a vector $e_{a}^{h}(\lambda)$ of assets; so, at $\lambda=0$, each household just consumes his endowment and no economic activity would take place. This treatment will be useful in proving that the equilibrium of $\mathscr{E}(0)$ is a regular zero point of the system (5.1), as is to be shown in Proposition 5.1.

We shall next characterize the equilibrium of $\mathscr{E}(\lambda)$ by a system of simultaneous equations, which would facilitate its computation. Let $D_{s}=\left(D_{s}^{1}, \cdots, D_{s}^{J}\right)$ for all $s \in$ $\mathbf{S}, Y=\left\{p, q,\left(\eta^{h}, \mu^{h}\right)_{h \in \mathbf{H}}\right\}, v^{h}=\left(v_{0}^{h}, \cdots, v_{S}^{h}\right)$, where $v_{0}^{h}=p_{0} e_{0}^{h}(\lambda)+(1-\lambda) q e_{a}^{h}(\lambda)$ and $v_{s}^{h}=p_{s} e_{s}^{h}(\lambda)-\lambda(1-\lambda) e_{a}^{h}(\lambda) D_{s}, \forall s \in \mathbf{S}$. Let

$$
\Omega_{\lambda}=\left[\begin{array}{cccccc}
p_{0} & 0 & \cdots & 0 & q & (1-2 \lambda) q \\
-\lambda \kappa_{1} \circ p_{1} & p_{1} & \cdots & 0 & -\lambda D_{1} & \lambda D_{1} \\
\vdots & \vdots & & \vdots & \vdots & \vdots \\
-\lambda \kappa_{S} \circ p_{S} & 0 & \cdots & p_{S} & -\lambda D_{S} & \lambda D_{S}
\end{array}\right]
$$

Since $U^{h}(\eta ; \lambda)$ is strictly concave in $\eta$ for any given $\lambda \in[0,1), Y$ is an equilibrium 
of $\mathscr{E}(\lambda)$ if and only if it solves the following system

$$
\left\{\begin{array}{l}
\nabla_{\eta} U^{h}-\Omega_{\lambda}^{T} \mu^{h}=0, h \in \mathbf{H} \\
\Omega_{\lambda} \eta^{h}-v^{h}=0, h \in \mathbf{H} \\
\sum_{h \in \mathbf{H}}\left[x_{0 l}^{h}-e_{0 l}^{h}(\lambda)\right]=0, l=1, \cdots, L-1 \\
\sum_{h \in \mathbf{H}}\left[x_{0 L}^{h}-e_{0 L}^{h}(\lambda)\right]+\lambda\left(\sum_{l \in \mathbf{L}} p_{0 l}-\zeta_{0}\right)=0 \\
\sum_{h \in \mathbf{H}}\left[x_{s l}^{h}-e_{s l}^{h}(\lambda)-\lambda F_{s l}\left(x_{0}^{h}\right)\right]=0, l=1, \cdots, L-1 \\
\sum_{h \in \mathbf{H}}\left[x_{s L}^{h}-e_{s L}^{h}(\lambda)-\lambda F_{s 1}\left(x_{0}^{h}\right)\right]+\lambda\left(\sum_{l \in \mathbf{L}} p_{s l}-\zeta_{s}\right)=0 \\
\sum_{h \in \mathbf{H}}\left[\lambda\left(\varphi^{h}-\psi^{h}\right)+(1-\lambda)\left(\varphi^{h}+\psi^{h}-e_{a}^{h}(\lambda)\right)\right]=0
\end{array}\right.
$$

where $\zeta=\left(\zeta_{0}, \cdots, \zeta_{S}\right) \in \mathscr{R}_{++}^{S+1}$. The first set of equations is made up of the KKT conditions of each household; the second of their budget constraints; the third and the fifth of commodity market clearing conditions; the last of asset market clearing conditions. For the fourth and sixth sets of equations, they reduce to the market clearing conditions when $\lambda$ vanishes; when it does not, their first terms are, by Walras' law, automatically zero and then they reduce to

$$
\sum_{l} p_{s l}=\zeta_{s}, \forall s \in \overline{\mathbf{S}} .
$$

(This formulation helps to prove Propositions 5.1 and 5.2.) Let $H(Y, \lambda)$ be the left hand side of (5.1). Define

$$
\begin{aligned}
& \Delta=\left\{(p, q) \in \mathscr{R}_{++}^{(S+1) L} \times \mathscr{R}_{++}^{J}:\left\|p_{s}\right\|_{1}=1, \forall s \in \mathbf{S}\right\}, \\
& X=\Delta \times \mathscr{R}_{++}^{N} \times \mathscr{R}_{+}, \quad y=\mathscr{R}^{N+(S+1) L+J},
\end{aligned}
$$

where $N=\hbar[(S+1)(L+1)+2 J]$. Then $H(Y, \lambda)$ is a Homotopy from $X$ to $y$.

\subsection{A path-following algorithm}

We shall next try to find the equilibrium of $\mathscr{E}$ by solving $H(Y, \lambda)=0$ through a path-following algorithm. To enable this, we need a primary route that emanates from $H(Y, 0)=0$ and heads for $H(Y, 1)=0$. Since $u^{h}(x)$ is smooth, the Homotopy $H$ is then smooth and, as a rule, we shall next show that $H(Y, 0)$ has a unique zero point, $H(Y, \lambda)$ has zero as its regular value for all $\lambda \in[0,1)$ and the set $\{(Y, \lambda) \in$ $X: H(Y, \lambda)=0\}$ is compact. 
Note that $H(Y, 0)$ is a characterization of the economy $\mathscr{E}(0)$, which, as stated above, has a unique equilibrium, so solution to $H(Y, 0)=0$ is unique. For its regularity, we claim that

Proposition 5.1. If all commodities are normal, then $H(Y, 0)$ has zero as its regular value.

Proof. Let $G_{1}$ be the first two rows (in block) of $G(Y, \lambda)$, or the first-order condition of each household, and $G_{2}$ be the rest. The Jacobian matrix $J_{0}$ of $H(Y, 0)$ with respect to $Y$ and $\left(\mu^{h}\right)$ is given by

$$
J_{0}=\left[\begin{array}{cccc:c}
G_{11}^{1} & & & & G_{12}^{1} \\
& G_{11}^{2} & & & G_{12}^{2} \\
& & \ddots & & \vdots \\
& & & G_{11}^{\hbar} & G_{12}^{\hbar} \\
\hdashline G_{21}^{1} & G_{21}^{2} & \cdots & G_{21}^{\hbar} & 0
\end{array}\right],
$$

where the upper left block is the Jacobian of $G_{1}$ with respect to $\left(\eta^{h}, \mu^{h}\right)_{h \in \mathbf{H}}$, and upper right to $(p, q)$; the lower left one is that of $G_{2}$ to $\left(\eta^{h}, \mu^{h}\right)_{h \in \mathbf{H}}$ and lower right to $(p, q)$. The blocks are each given by, letting $I_{n}$ be the identity matrix of size $n$,

$$
G_{11}^{h}=\left[\begin{array}{cc}
\nabla_{\eta} U^{h} & -\Omega_{0}^{T} \\
\Omega_{0} & 0
\end{array}\right], G_{21}^{h}=\left[\begin{array}{cccc}
I_{(S+1) L} & 0 & 0 & 0 \\
0 & I_{J} & I_{J} & 0
\end{array}\right],
$$

and,

$$
G_{12}^{h}=\left[\begin{array}{cc}
\Pi^{h} & 0 \\
0 & -\mu_{0}^{h} I_{J} \\
0 & -\mu_{0}^{h} I_{J} \\
0 & 0
\end{array}\right], \Pi^{h}=\left[\begin{array}{ccc}
-\mu_{0}^{h} I_{L} & & \\
& \ddots & \\
& & -\mu_{S}^{h} I_{L}
\end{array}\right] .
$$

That household $h$ precisely consumes his endowment makes the last row of $G_{12}^{h}$ vanish. Letting

$$
\Theta^{h}=\left[\begin{array}{ll}
\Pi^{h} & \\
& -\mu_{0}^{h}
\end{array}\right],
$$

we have $G_{12}^{h}=\left(G_{21}^{h}\right)^{T} \Theta^{h}$. Recall that $\mu_{s}^{h}$ is the shadow price. By assuming that all commodities are normal, we get $\mu_{s}^{h}>0$ for all $s \in \overline{\mathbf{S}}, h \in \mathbf{H}$. The matrix $\Theta^{h}$ is thus diagonal and negative definite. Since all $G_{11}^{h}$ are negative definite, by elementary 
column operation, $J_{0}$ is reduced to

$$
\left[\begin{array}{ccccc}
G_{11}^{1} & & & & 0 \\
& G_{11}^{2} & & & 0 \\
& & \ddots & & \vdots \\
& & & G_{11}^{\hbar} & 0 \\
G_{21}^{1} & G_{21}^{2} & \cdots & G_{21}^{\hbar} & G_{22}
\end{array}\right],
$$

where $G_{22}=-\sum_{h} G_{21}^{h}\left(G_{11}^{h}\right)^{-1} G_{12}^{h}=-\sum_{h} G_{21}^{h}\left(G_{11}^{h}\right)^{-1}\left(G_{21}^{h}\right)^{T} \Theta^{h}$. The matrix $G_{21}^{h}\left(G_{11}^{h}\right)^{-1}\left(G_{21}^{h}\right)^{T}$ is negative definite, for $\left(G_{11}^{h}\right)^{-1}$ is negative definite and $G_{21}^{h}$ is of full row rank. Hence, the matrix $-G_{21}^{h}\left(G_{11}^{h}\right)^{-1}\left(G_{21}^{h}\right)^{T} \Theta^{h}$, and therefore $G_{22}$, is negative definite. Thus $J_{0}$ is nonsingular.

$$
\text { Let } \xi=\left(\xi_{1}, \cdots, \xi_{L+\hbar}\right)=\left(e_{1}^{1}, \cdots, e_{L}^{1}, e_{1}^{2}, e_{1}^{3}, \cdots, e_{1}^{\hbar}, \zeta\right) \in \mathscr{R}^{L+\hbar} \text {. We have }
$$

LEMMA 5.1. When $p \gg 0$, the Jacobian of the following system with respect to $\xi$ is nonsingular:

$$
\begin{aligned}
& p\left(x^{h}-e^{h}\right)=0, h \in \mathbf{H} \\
& \sum_{h \in \mathbf{H}}\left(x_{l}^{h}-e_{l}^{h}\right)=0, l=1, \cdots, L-1 \\
& \sum_{h \in \mathbf{H}}\left(x_{L}^{h}-e_{L}^{h}\right)+\sum_{l} p_{l}-\zeta=0,
\end{aligned}
$$

where $x^{h}, e^{h}$ and $p$ all belong to $\mathscr{R}^{L}$.

PROOF. The Jacobian $J$ is given by

$$
\left[\begin{array}{cccc:ccc:c}
p_{1} & p_{2} & \cdots & p_{L} & & & & 0 \\
\hdashline & & & & p_{1} & & & 0 \\
& & & & & \ddots & & \vdots \\
& & & & & & p_{1} & 0 \\
\hdashline 1 & 0 & \cdots & 0 & 1 & \cdots & 1 & 0 \\
0 & 1 & \cdots & 0 & 0 & \cdots & 0 & 0 \\
& & \ddots & & & \ddots & & \vdots \\
0 & 0 & \cdots & 1 & 0 & \cdots & 0 & 1
\end{array}\right] .
$$

It suffices to show that the system $J z=0$ has zero as its unique solution. In fact, for any $z$ satisfying $J z=0$, by $p_{1}>0$, it follows from the second to the $L$-th equation that $z_{i}=0, i=L+1, \cdots, L+\hbar-1$, which, along with the $(L+1)$-th equation, implies $z_{1}=0$. From $(L+2)$-th to the $(L+\hbar-1)$-th equation it follows that $z_{i}=$ $0, i=2, \cdots, L-1$, which, along with the first equation and $p_{L}>0$, implies $z_{L}=0$. By the last equation, we then have $z_{L+\hbar}=0$. So $z=0$ and $J$ is thus nonsingular. 
Remark 1. In this Lemma, the first set of equations corresponds to households' budget constraints in some state, the second to the market clearing condition; and the third shapes the price simplex. If we replace the last column of $J$ with the vector $(0, \cdots, 0, \lambda)$, still it is nonsingular when $\lambda \neq 0$.

Remark 2. Let $\xi_{0}=\left(e_{01}^{1}, \cdots, e_{0 L}^{1},(1-\lambda) e_{a 1}^{1}, \cdots,(1-\lambda) e_{a J}^{1}, e_{01}^{2},, e_{01}^{3}, \cdots, e_{01}^{\hbar}, \zeta_{0}\right) \in$ $\mathscr{R}^{L+\hbar+J}$. The system in state 0 that corresponds to system (5.2), is given by

$$
\begin{aligned}
& p_{0}\left(x_{0}^{h}-e_{0}^{h}\right)+\lambda q\left(\varphi^{h}-\psi^{h}\right)+(1-\lambda) q\left(\varphi^{h}+\psi^{h}-e_{a}^{h}\right)=0, h \in \mathbf{H} \\
& \sum_{h \in \mathbf{H}}\left[x_{0 l}^{h}-e_{0 l}^{h}(\lambda)\right]=0, l=1, \cdots, L-1, \\
& \sum_{h \in \mathbf{H}}\left[x_{0 L}^{h}-e_{0 L}^{h}(\lambda)\right]+\lambda\left(\sum_{l} p_{0 l}-\zeta_{0}\right)=0, \\
& \sum_{h \in \mathbf{H}}\left[\lambda\left(\varphi^{h}-\psi^{h}\right)+(1-\lambda)\left(\varphi^{h}+\psi^{h}-e_{a}^{h}(\lambda)\right)\right]=0 .
\end{aligned}
$$

By Lemma 5.1, the Jacobian $J_{0}$ of this system with respect to $\xi_{0}$, is nonsingular. Similarly, letting $\xi_{s}=\left(e_{s 1}^{1}, \cdots, e_{s L}^{1}, e_{s 1}^{2},, e_{s 1}^{3}, \cdots, e_{s 1}^{\hbar}, \zeta_{s}\right) \in \mathscr{R}^{L+\hbar}, \forall s \in \mathbf{S}$, the Jacobian $J_{s}$ of the system in state $s$ with respect to $\xi_{s}$ is also nonsingular.

$$
\text { Let } \sigma=\left(\sigma^{1}, \cdots, \sigma^{\hbar}, \xi_{0}, \cdots, \xi_{S}\right) \text {. We have }
$$

Proposition 5.2. When $\lambda \in(0,1), H(Y, \lambda)$ has zero as its regular value for almost all $\sigma \in y_{++}$.

Proof. The Jacobian of $H$ with respect to $\sigma$ is given by

$$
J_{\sigma} H=\left[\begin{array}{cccccc}
\Lambda & & & & & \\
& \ddots & & & & \\
& & \Lambda & & & \\
& & & J_{0} & & \\
& & & & \ddots & \\
& & & & & J_{S}
\end{array}\right],
$$

where

$$
\Lambda=\left[\begin{array}{lll}
\lambda(1-\lambda) & & \\
& \ddots & \\
& & \lambda(1-\lambda)
\end{array}\right]
$$

So $J_{\sigma} H$ is nonsingular when $\lambda \in(0,1)$. The Jacobian of $H: X \times y_{++} \rightarrow y$, taken $\sigma$ as variables, then has full row rank. Noting that $H$ is smooth, the proposition follows immediately from the transversality theorem.

Q.E.D 
Propositions 5.1 and 5.2 together imply that the primary route desired exists. We now dedicate ourselves to the proposition:

Proposition 5.3. For any $\varepsilon>0$, the primary route defined by $H(Y, \lambda)=0$ is trapped when $\lambda \in[0,1-\varepsilon]$.

ProOF. From the proof of Proposition 4.1, it follows that $\left\|\eta^{h}\right\|$ is finite for all $h \in \mathbf{H}$, and the commodity price vector $p$ and the asset price vector $q$ are strictly positive and of finite magnitude. So it remains to show that $\left\|\mu^{h}\right\|$ is finite. The first-order condition of household $h$ is given by

$$
\begin{aligned}
& \frac{\partial U^{h}}{\partial \varphi_{j}}=\mu_{0}^{h} q_{j}, \\
& \frac{\partial U^{h}}{\partial x_{s l}}=\mu_{s}^{h} p_{s l}, \forall s \in \mathbf{S} .
\end{aligned}
$$

Because $U^{h}$ is smooth, partial derivatives on the left-hand side are continuous on a compact set; so their function values are bounded, from which it follows immediately that $\left\|\mu^{h}\right\|$ is finite.

Q.E.D

\section{Numerical Test}

Let $\bar{e}_{s l}$ be the reciprocal of $e_{s l}$. The starting point of the algorithm, or the equilibrium of economy $\mathscr{E}(0)$, is given by: (i) the equilibrium prices of commodities and of assets

$$
p_{s l}=\frac{\bar{e}_{s l}}{\sum_{k=1}^{L} \bar{e}_{s k}}, \forall s \in \overline{\mathbf{S}}, l \in \mathbf{L} ; \quad q_{j}=\frac{2 p_{01} e_{01}}{\sum_{h} e_{a j}^{h}}, \forall j \in \mathbf{J}
$$

(ii) the equilibrium consumption of commodities and of assets in state 0

$$
\begin{aligned}
& x_{0 l}^{h}=\frac{1}{(L+2 J) p_{0 l}}\left(\sum_{k=1}^{L} p_{0 k} e_{0 k}^{h}+\sum_{j=1}^{J} q_{j} e_{a j}^{h}\right), \forall l \in \mathbf{L} ; \\
& \varphi_{j}^{h}=\psi_{j}^{h}=\frac{1}{(L+2 J) q_{j}}\left(\sum_{k=1}^{L} p_{0 k} e_{0 k}^{h}+\sum_{j=1}^{J} q_{j} e_{a j}^{h}\right), \forall j \in \mathbf{J} ;
\end{aligned}
$$

(iii) the equilibrium consumption in state $s$

$$
x_{s l}^{h}=\frac{1}{L \cdot p_{s l}} \sum_{k=1}^{L} p_{s k} e_{s k}^{h}, \forall s \in \mathbf{S}, l \in \mathbf{L} .
$$


To test the algorithm, we adopt the classical path-following algorithm with Euler predictor and Newton corrector (Allgower and Georg, 1993), and program it in MATLAB. The pseudocode for the implementation of this algorithm is given in the Appendix. We take the step size in the predictor step to be 0.25 , and let $\lambda_{0}=0.2, e_{a}^{h}=1, h \in \mathbf{H}$.

EXAMPLE 1. First we use the algorithm presented in the last section to find a solution to the example given in Section 3, and compare it with the true equilibrium. Let $\left(\gamma_{11}, \gamma_{12}\right)=\left(\gamma_{21}, \gamma_{22}\right)=(0.95,0.05)$. If the collateral requirement level is at $1 / 4$, the equilibrium resulting from the algorithm is given by

$$
\begin{aligned}
q & =0.9533, p=(1,6.5189 ; 1,3.9777 ; 1,3.0756), \\
x^{1} & =(17.3173,0.0009 ; 12.8898,0.0003 ; 11.4512,0.1945), \\
x^{2} & =(0.6811,0.9929 ; 5.1105,0.9774 ; 0.0487,0.7885) ;
\end{aligned}
$$

if at $1 / 3$, by

$$
\begin{aligned}
q & =0.9881, p=(1,5.8813 ; 1,3.9856 ; 1,3.1321), \\
x^{1} & =(17.4043,0.0014 ; 11.9720,0.0003 ; 11.4486,0.1610), \\
x^{2} & =(0.5966,0.9835 ; 6.0280,0.9782 ; 0.0513,0.8168) ;
\end{aligned}
$$

and at $1 / 2$, by

$$
\begin{aligned}
q & =0.9839, p=(1,5.0622 ; 1,3.9893 ; 1,3.8779), \\
x^{1} & =(17.4809,0.0096 ; 10.9678,0.0003 ; 10.8702,0.0182), \\
x^{2} & =(0.5235,0.9378 ; 7.0322,0.9442 ; 0.6298,0.9256) .
\end{aligned}
$$

It is easily checked that these three equilibria are all in the neighborhood of the true equilibria given in Section 3.

EXAMPLE 2. Suppose each household has a CES utility function which is additively separable across states. That is, for household $h$ and $\tau>0$,

$$
u^{h}(x)=u^{h}\left(x_{0}, \cdots, x_{S}\right)=\sum_{s=0}^{S} \gamma_{s}^{h}\left(\sum_{\ell=1}^{L} x_{s \ell}^{h \tau}\right)^{\frac{1}{h \tau}},
$$

where $\gamma_{s}^{h}$ is the probability of state $s$ with $\gamma_{0}^{h}=1$. In the real economic world, both the endowments of the households and the payoffs of the assets may be affected by some random factors. For this reason, let us suppose that the vectors $e^{h}, A^{j}, c^{j}$ are randomly generated for all households and all assets. (Their values are given in the Appendix.) Let $S=5, L=3, \hbar=4, J=2$, and $\tau=0.2$. 
The collateral equilibrium of this economy yielded by the algorithm is the following. The asset prices are given by

$$
q=\left[\begin{array}{ll}
q_{1} & q_{2}
\end{array}\right]=\left[\begin{array}{ll}
1.5412 & 1.6338
\end{array}\right] .
$$

The commodity prices are given by

$$
p=\left[p_{0}, \cdots, p_{S}\right]=\left[\begin{array}{llllll}
1.0000 & 1.0000 & 1.0000 & 1.0000 & 1.0000 & 1.0000 \\
1.2867 & 0.9004 & 0.8089 & 1.0144 & 0.7211 & 0.7525 \\
0.9675 & 1.1813 & 0.7611 & 1.0618 & 0.6203 & 0.7035
\end{array}\right]
$$

The consumption bundles of the households are given by

$$
\begin{aligned}
x^{1} & =\left[\begin{array}{cccccc}
30.7869 & 9.7293 & 15.4899 & 33.1254 & 7.9715 & 14.4002 \\
8.9621 & 15.2138 & 42.6964 & 30.8866 & 36.3267 & 51.2627 \\
45.3779 & 5.0494 & 57.5430 & 25.0778 & 75.2209 & 70.4086
\end{array}\right] ; \\
x^{2} & =\left[\begin{array}{llllll}
48.1652 & 45.7008 & 28.2635 & 63.5420 & 20.5380 & 28.8742 \\
21.6899 & 58.7556 & 46.5104 & 61.2135 & 45.0108 & 57.2464 \\
55.4136 & 30.8510 & 53.7632 & 54.7020 & 64.7442 & 67.4191
\end{array}\right] ; \\
x^{3} & =\left[\begin{array}{cccccc}
15.9227 & 38.3039 & 16.0060 & 38.6115 & 21.2755 & 26.6751 \\
9.4738 & 45.4373 & 22.5129 & 37.6881 & 35.4247 & 41.1774 \\
19.7094 & 29.2801 & 24.8420 & 35.0035 & 44.7285 & 45.5600
\end{array}\right] ; \\
x^{4} & =\left[\begin{array}{llllll}
36.1251 & 26.1850 & 37.9519 & 49.8375 & 21.0008 & 27.5178 \\
26.8742 & 29.7540 & 49.2010 & 48.9647 & 31.2117 & 38.2462 \\
39.4992 & 21.4048 & 52.9939 & 46.3467 & 37.3232 & 41.1673
\end{array}\right] ;
\end{aligned}
$$

where the $s$-th column of $x^{h}$ denotes the consumption bundle of household $h$ in state $s-1$. The purchase portfolio of assets are given by

$$
\varphi=\left[\varphi^{1}, \cdots, \varphi^{\hbar}\right]=\left[\begin{array}{cccc}
0.2660 & 16.1148 & 0.3098 & 5.7091 \\
9.5097 & 1.6288 & 0.3141 & 6.4711
\end{array}\right]
$$

The sales portfolio of assets are given by

$$
\psi=\left[\psi^{1}, \cdots, \psi^{\hbar}\right]=\left[\begin{array}{cccc}
10.0625 & 2.4305 & 4.7438 & 5.5636 \\
1.4598 & 10.4798 & 2.3015 & 3.9953
\end{array}\right] .
$$

EXAMPLE 3. Suppose the utility function takes the same form as in Example 2. Let $S=6, L=5, \hbar=6, J=5$. The values of $e^{h}, A^{j}, c^{j}$ for all households and all assets are given in the Appendix. 
The collateral equilibrium of this economy yielded by the algorithm is the following. The asset prices are given by

$$
q=\left[q_{1}, \cdots, q_{5}\right]=\left[\begin{array}{lllll}
3.6225 & 4.6209 & 4.6142 & 3.0501 & 0.5184
\end{array}\right] .
$$

The commodity prices are given by

$$
p=\left[p_{0}, \cdots, p_{S}\right]=\left[\begin{array}{lllllll}
1.0000 & 1.0000 & 1.0000 & 1.0000 & 1.0000 & 1.0000 & 1.0000 \\
1.6196 & 1.1545 & 0.9862 & 1.0053 & 0.8357 & 0.7216 & 1.1916 \\
1.3175 & 1.0238 & 0.7775 & 0.8552 & 0.7698 & 0.6728 & 0.5463 \\
1.3786 & 1.0187 & 0.8842 & 0.8070 & 1.3453 & 0.6883 & 0.6724 \\
1.0847 & 1.2917 & 1.1307 & 1.0308 & 0.8468 & 0.6397 & 0.7629
\end{array}\right] .
$$

The consumption bundles of the households are given by

$$
\begin{aligned}
x^{1} & =\left[\begin{array}{lllllll}
31.7171 & 39.5299 & 21.5848 & 29.2675 & 23.7285 & 7.2628 & 8.8528 \\
4.9991 & 20.2420 & 23.0405 & 28.6000 & 54.6414 & 32.5385 & 4.8371 \\
24.5323 & 35.3583 & 72.0056 & 58.4940 & 80.8021 & 45.5803 & 120.7635 \\
9.6012 & 36.2077 & 38.6161 & 76.6042 & 6.7764 & 40.7900 & 45.8896 \\
26.4623 & 12.2088 & 12.3009 & 25.6913 & 51.2922 & 58.0997 & 26.1317
\end{array}\right] \\
x^{2} & =\left[\begin{array}{lllllll}
38.5556 & 44.2165 & 29.0851 & 39.9266 & 21.0486 & 20.0233 & 9.7375 \\
11.6075 & 31.2951 & 30.0478 & 39.4095 & 31.5355 & 42.4148 & 6.5546 \\
28.7847 & 41.7645 & 52.4802 & 58.3657 & 38.0389 & 50.0593 & 40.1668 \\
17.3758 & 42.2757 & 38.8009 & 67.3477 & 10.9972 & 47.4018 & 24.5211 \\
22.9607 & 23.9272 & 21.8808 & 37.0827 & 30.6190 & 56.3886 & 18.1935
\end{array}\right] \\
x^{3} & =\left[\begin{array}{lllllll}
32.9370 & 47.7945 & 22.0065 & 26.1771 & 20.6505 & 26.1306 & 20.7563 \\
14.9927 & 37.8119 & 22.4934 & 25.9489 & 27.3739 & 43.7408 & 15.9471 \\
27.6627 & 45.9831 & 32.5242 & 33.5834 & 31.1551 & 48.9799 & 52.1707 \\
18.8922 & 46.3650 & 26.6831 & 36.8832 & 12.9727 & 47.1878 & 37.9736 \\
24.4328 & 31.4999 & 18.1698 & 24.9258 & 26.8245 & 53.1157 & 31.3233
\end{array}\right] \\
x^{4} & =\left[\begin{array}{lllllll}
22.2125 & 32.9438 & 43.2533 & 24.8424 & 24.7025 & 19.2178 & 19.3384 \\
12.7670 & 27.5694 & 43.9896 & 24.6714 & 30.4619 & 28.5571 & 15.6510 \\
21.8966 & 31.9860 & 58.2903 & 30.0105 & 33.5481 & 31.1539 & 40.0951 \\
16.7915 & 32.1881 & 50.1580 & 32.2159 & 17.3575 & 30.2743 & 31.2411 \\
19.6309 & 24.0551 & 37.3004 & 23.9329 & 30.0197 & 33.1636 & 26.8333
\end{array}\right] \\
x^{5}= & {\left[\begin{array}{lllllll}
35.8255 & 65.1494 & 31.4687 & 38.8099 & 35.0259 & 47.0290 & 29.5189 \\
24.0390 & 56.0039 & 31.9075 & 38.5681 & 41.7224 & 65.0201 & 24.7648 \\
37.0932 & 63.5290 & 39.9583 & 45.5137 & 45.2327 & 69.9266 & 53.4455 \\
28.6254 & 63.8707 & 35.4997 & 48.2955 & 26.0138 & 68.2649 & 43.7721 \\
32.1391 & 50.0370 & 27.8727 & 37.6132 & 41.2459 & 73.6452 & 38.8057
\end{array}\right] }
\end{aligned}
$$




$$
x^{6}=\left[\begin{array}{lllllll}
22.7523 & 35.8134 & 27.6008 & 39.3881 & 25.5936 & 28.0723 & 31.7188 \\
15.5947 & 31.2587 & 27.9330 & 39.1544 & 29.7737 & 37.3217 & 27.1820 \\
24.0304 & 35.0066 & 33.5601 & 45.5639 & 31.9516 & 39.9344 & 53.7352 \\
18.7139 & 35.1783 & 30.6491 & 48.0985 & 19.6488 & 39.0440 & 44.9446 \\
20.3743 & 28.2949 & 24.8359 & 38.2657 & 29.5002 & 41.8989 & 40.3416
\end{array}\right]
$$

where the $s$-th column of $x^{h}$ denotes the consumption bundle of household $h$ in state $s-1$. The purchase portfolio of assets are given by

$$
\varphi=\left[\varphi^{1}, \cdots, \varphi^{\hbar}\right]=\left[\begin{array}{cccccc}
0.6782 & 1.4088 & 0.9744 & 0.7940 & 2.2582 & 1.6817 \\
0.7588 & 0.4364 & 0.8550 & 0.5524 & 2.6283 & 1.4551 \\
0.6666 & 0.4570 & 0.7650 & 0.5510 & 2.5761 & 1.4375 \\
1.3760 & 0.7798 & 0.7779 & 0.8837 & 3.2208 & 1.9534 \\
2.5054 & 5.5671 & 7.7062 & 4.3951 & 28.9272 & 14.4902
\end{array}\right]
$$

The sales portfolio of assets are given by

$$
\psi=\left[\psi^{1}, \cdots, \psi^{\hbar}\right]=\left[\begin{array}{cccccc}
0.7436 & 0.8544 & 1.2878 & 1.3894 & 2.2781 & 1.3399 \\
0.3898 & 1.6719 & 1.2108 & 1.0777 & 1.4403 & 0.9705 \\
0.3970 & 1.4192 & 1.2415 & 1.0402 & 1.4451 & 0.9804 \\
0.4303 & 1.2742 & 2.0993 & 1.4609 & 2.2604 & 1.5885 \\
17.3786 & 9.8569 & 9.7024 & 7.8093 & 12.0200 & 8.0574
\end{array}\right]
$$

For Examples 2 and 3, we can not compare directly the equilibria given above with the true equilibria, which are unknown. But it can be checked that both equilibria yielded by the algorithm satisfy system (5.1), so we can conclude that they are in the neighborhood of the true equilibria.

\section{Appendix}

\section{Proof of Theorem 4.1}

It is noticed that the budget set $\tilde{B}^{h}(p, q, \lambda)$ is unbounded. The key idea of the proof is first to impose, to make $\tilde{B}^{h}(p, q, \lambda)$ bounded, lower and upper bounds on the asset prices and on one's consumption, and then let the lower bounds go to zero and the upper bounds go to infinity. The technique of proof used here is analogous to that of Dubey et al. (2005).

For any small lower bound $b>0$, define

$$
\Delta_{b}=\left\{(p, q) \in \mathscr{R}_{+}^{(S+1) L} \times \mathscr{R}_{+}^{J}:\left\|p_{s}\right\|_{1}=1 \forall s \in \overline{\mathbf{S}}, p \geq b \text {, and } b \leq q \leq \frac{1}{b}\right\} .
$$


It is noticed that when $\|x\| \rightarrow \infty,\left\|U^{h}(x)\right\| \rightarrow \infty$, so there exists an $M>\|2 e\|$ such that when $\|x\|>M, U^{h}(x)>U^{h}(2 e)$ for all $h \in \mathbf{H}$. Define, for each $h \in \mathbf{H}$ and $(p, q) \in \Delta_{b}$,

$$
\begin{gathered}
\square^{h}=\left\{(x, \varphi, \psi) \in \mathscr{R}_{+}^{(S+1) L} \times \mathscr{R}_{+}^{J} \times \mathscr{R}_{+}^{J}:\|x\| \leq M\right\}, \\
\Psi_{b}^{h}(p, q)=\underset{(x, \varphi, \psi)}{\operatorname{argmax}}\left\{U^{h}(\eta ; \lambda):(x, \varphi, \psi) \in \tilde{B}^{h}(p, q, \lambda) \cap \square^{h}\right\} .
\end{gathered}
$$

We claim that $\Psi_{b}^{h}(p, q)$ is nonempty, compact, convex, and upper semi-continuous. Obviously, $\tilde{\tilde{B}}^{h}(p, q, \lambda) \cap \square^{h}$ is nonempty, convex, and closed. We next show its boundedness. Let $e_{a}=\sum_{h} e_{a}^{h}$. When $\lambda \in[0,0.25]$, we have, by (4.1),

$$
\|\varphi\| \leq M_{1} ; \quad\|\psi\| \leq M_{1}
$$

where $M_{1}=\frac{2}{b^{2}}\left\|e_{a}\right\|_{1}+\frac{2}{b}\left\|e_{0}\right\|_{1}$. When $\lambda \in[0.25,1)$, we have, by $\left(2.2^{\prime}\right)$ and $c^{j}>0$,

$$
\psi_{j} \leq \frac{4\left\|x_{0}\right\|}{\left\|c^{j}\right\|} \leq \frac{4 M}{\left\|c^{j}\right\|} .
$$

Let $\varsigma_{0}=\max _{j \in \mathbf{J}} 1 /\left\|c_{j}\right\|$ and $\varsigma=4 \varsigma_{0}$, then $\|\psi\| \leq \varsigma M$. By (2.1), we have

$$
\|\varphi\| \leq \frac{\left\|e_{0}^{h}\right\|_{1}}{b}+\frac{1+\varsigma J}{b^{2}}\left(M+\left\|e_{a}^{h}\right\|_{1}\right) \stackrel{\text { def }}{=} M_{2} .
$$

Let $K_{1}=\max \left(M_{1}, M_{2}\right)$ and $K_{2}=\max \left(M_{1}, \varsigma M\right)$. So, for all $\lambda \in[0,1),\|\varphi\| \leq K_{1}$ and $\|\psi\| \leq K_{2}$. Hence $\tilde{\tilde{B}}^{h}(p, q, \lambda) \cap \square^{h}$ is bounded. Consider the convex program

$$
\mathcal{M}=\underset{(x, \varphi, \psi)}{\operatorname{argmax}}\left\{\bar{u}^{h}(\eta ; \lambda):(x, \varphi, \psi) \in \tilde{\tilde{B}}^{h}(p, q, \lambda) \cap \square^{h}\right\} .
$$

Because of the continuity of $\bar{u}^{h}, \mathcal{M}$ is nonempty and bounded (it is a subset of $\left.\tilde{\tilde{B}}^{h}(p, q, \lambda) \cap \square^{h}\right)$. Then Theorem 5 of Wright (1992) applies, from which it follows that $\Psi_{b}^{h}(p, q)$ is nonempty, compact, and convex. For its upper semi-continuity, refer to section 1.8 of Debreu (1959).

Define the excess demand of commodities and of assets as

$$
\begin{aligned}
& Z_{0}=\sum_{h}\left(x_{0}^{h}-e_{0}^{h}\right), \\
& Z_{s}=\sum_{h}\left[x_{s}^{h}-e_{s}^{h}-\lambda F_{s}\left(x_{0}^{h}\right)\right], \forall s \in \mathbf{S} ; \\
& Z_{a}=\sum_{h \in \mathbf{H}}\left[\lambda\left(\varphi^{h}-\psi^{h}\right)+(1-\lambda)\left(\varphi^{h}+\psi^{h}-e_{a}^{h}\right)\right] .
\end{aligned}
$$


Write $Z=\left(Z_{0}, \cdots, Z_{S}, Z_{a}\right)$. Define the correspondence $\Psi_{b}^{0}$ to be

$$
\Psi_{b}^{0}(Z)=\underset{(p, q) \in \Delta_{b}}{\operatorname{argmax}}\left\{\sum_{s \in \overline{\mathbf{S}}} p_{s} \cdot Z_{s}+q \cdot Z_{a}\right\},
$$

which is clearly nonempty, compact, convex, and upper semi-continuous. Let $\square^{H}=\prod_{h \in \mathbf{H}} \square^{h}$, and $\Psi_{b}(p, q, Z): \Delta_{b} \times \square^{H} \rightarrow \Delta_{b} \times \square^{H}$ be the correspondence defined by

$$
\Psi_{b}(p, q, Z)=\Psi_{b}^{0}(Z) \times\left(\prod_{h \in \mathbf{H}} \Psi_{b}^{h}(p, q)\right) .
$$

By Kakutani's theorem, it has a fixed point $\left\{p(b), q(b),\left(x^{h}(b), \varphi^{h}(b), \psi^{h}(b)\right)_{h \in \mathbf{H}}\right\}$. As in Dubey et al. (2005), it can be established that, as $b$ tends to zero, this fixed point converges to an equilibrium of $\mathscr{E}(\lambda)$. To avoid notational clutter, we suppress the $b$.

In state 0 , we have $p_{0} Z_{0}+q Z_{a}=0$. If there is a $j \in \mathbf{J}$ such that $Z_{a j}>0$, taking $\tilde{q}_{j}=1 / b$ and $\tilde{q}_{i}=b$ for $i \neq j$, we have

$$
p_{0} Z_{0}+\tilde{q} Z_{a} \leq 0
$$

there then follows that,

$$
\frac{1}{b} Z_{a j} \leq\left\|e_{0}\right\|_{1}+b\left(\left\|e_{a}\right\|_{1}+J h K_{2}\right) \stackrel{\text { def }}{=} T_{1} .
$$

So we have, for all $j \in \mathbf{J}$,

$$
Z_{a j} \leq b T_{1}
$$

If there is an $l \in \mathbf{L}$ such that $Z_{0 l}>0$, taking $\tilde{p}_{0 l}=1-(L-1) b, \tilde{p}_{0 k}=b$ for all $k \neq l$, and $\tilde{q}_{j}=b$ for all $j \in \mathbf{J}$, we have

$$
Z_{0 l} \leq \frac{b}{1-(L-1) b}\left(\left\|e_{0}\right\|_{1}+J h K_{2}\right) \stackrel{\text { def }}{=} b T_{2} .
$$

This being true for all $l \in \mathbf{L}$, it follows,

$$
\left\|\sum_{h} F_{s}\left(x_{0}^{h}\right)\right\| \leq\left\|F_{S}\left(e_{0}\right)\right\|+b\left\|F_{s}\left(\mathbf{T}_{2}\right)\right\| \stackrel{\text { def }}{=} T_{3},
$$

where $\mathbf{T}_{2}=\left(T_{2}, \cdots, T_{2}\right) \in R^{L}$.

In state $s$, in view of the strict monotonicity of the utility function, we have

$$
p_{s}\left(x_{s}^{h}-e_{s}^{h}\right)-\lambda p_{s} F_{s}\left(x_{0}^{h}\right)+\sum_{j} \lambda\left(\psi_{j}^{h}-\varphi_{j}^{h}+(1-\lambda) e_{a}^{h}\right) \mathrm{DEL}_{s}^{j}=0 .
$$


Adding across households, we get

$$
p_{s} Z_{s}+\lambda \sum_{h} \sum_{j}\left(\psi_{j}^{h}-\varphi_{j}^{h}+(1-\lambda) e_{a}^{h}\right) \mathrm{DEL}_{s}^{j}=0 .
$$

Note that $\sum_{h}\left(\varphi_{j}^{h}-\psi_{j}^{h}-(1-\lambda) e_{a}^{h}\right) \leq Z_{a j}$ and $\operatorname{DEL}_{s}^{j} \leq p_{s} A_{s}^{j}$ for all $\lambda \in[0,1]$. If there exists an $l \in \mathbf{L}$ such that $Z_{s l}>0$, taking $\tilde{p}_{s l}=1-(L-1) b$, and $\tilde{p}_{s k}=b$ for all $k \neq l$, we obtain

$$
Z_{s l} \leq \frac{b}{1-(L-1) b}\left(\left\|e_{s}\right\|_{1}+L T_{3}+J T_{1} T_{4}\right)
$$

where $T_{4}=\max \left(\left\|A_{s}^{1}\right\|_{1}, \cdots,\left\|A_{s}^{J}\right\|_{1}\right)$. Therefore, aggregate excess demand of commodities and of assets becomes less than or equal to zero, as $b$ goes to zero. By the argument of Dubey et al. (2005) (p. 32), we have that commodity prices are all positive, asset prices are bounded, and there is no excess supply in any commodity and in any asset. Indeed, we have here that $q \gg 0$, i.e., asset prices all being positive.

The Pseudocode of the Algorithm

The pseudocode is presented in the MATLAB style. In the following the symbol, step, denotes the step length; step0, stepl denote respectively the minimum and the maximal step lengths allowable in the algorithm. The vector

$$
\text { start }=\left[\begin{array}{l}
Y \\
\lambda
\end{array}\right]
$$

denotes the starting point of each step, and tan designates for the Euler predictor the tangent vector; its definition and the procedure for its computation are given in Definition 2.2 and Section 3.2 of Allgower and Georg (1993). Let the symbol, err, be the error of the system, with 'start(end)' denoting the last component of start,

$$
\left\{\begin{array}{l}
H(Y, \lambda)=0 \\
\operatorname{start}(\text { end })-\lambda=0
\end{array}\right.
$$

at a certain point, and errO the maximal error allowed in each step. If err is greater than erro, the step length, step, will be contracted by a factor of 'contraction', until its value becomes less than stepo.

The pseudocode is sketched as follows. For details on the implementation of Euler predictor and Newton corrector, one is referred to Allgower and Georg (1993, 1994). 
(i) Set the values of step0, stepl, step, errO, err, contraction, delta. Let lambda=0.

(ii) Let start be the solution to the system (6.1) with $\lambda=0$. Take the initial tangent vector to be $\tan =[0, \cdots, 0,1]^{T}$.

(iii) WHILE lambda<1-delta

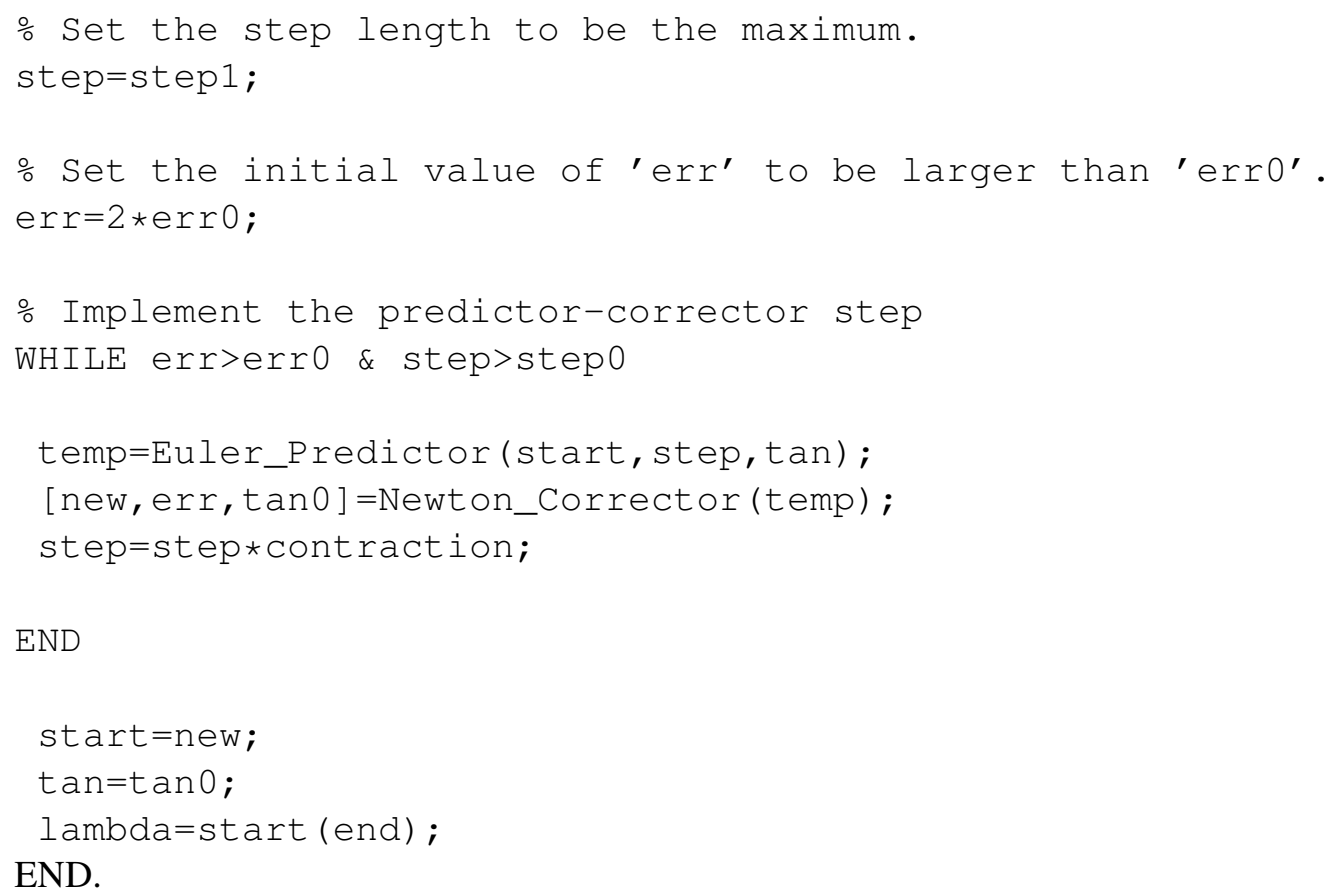

Data of Example 2

The endowments of the households are given by

$$
\begin{aligned}
& e^{1}=\left[\begin{array}{cccccc}
23 & 4 & 14 & 6 & 4 & 17 \\
11 & 1 & 18 & 29 & 47 & 45 \\
49 & 1 & 46 & 25 & 23 & 25
\end{array}\right] ; e^{2}=\left[\begin{array}{ccccccc}
48 & 22 & 39 & 3 & 15 & 1 \\
33 & 34 & 2 & 40 & 35 & 44 \\
48 & 27 & 50 & 32 & 35 & 32
\end{array}\right] ; \\
& e^{3}=\left[\begin{array}{ccccccc}
17 & 42 & 2 & 20 & 39 & 24 \\
2 & 49 & 46 & 33 & 8 & 41 \\
18 & 18 & 11 & 49 & 43 & 44
\end{array}\right] ; e^{4}=\left[\begin{array}{cccccc}
43 & 4 & 37 & 32 & 1 & 3 \\
21 & 17 & 46 & 32 & 40 & 44 \\
45 & 14 & 10 & 15 & 10 & 5
\end{array}\right] ;
\end{aligned}
$$

where the $s$-th column of $e^{h}$ denotes the endowment of household $h$ in state $s-1$. The durability of the commodities are given by

$$
\left[\kappa_{1}, \cdots, \kappa_{5}\right]=\left[\begin{array}{lllll}
0.3658 & 0.0436 & 0.9475 & 0.0900 & 0.4005 \\
0.7188 & 0.7302 & 0.6680 & 0.2683 & 0.2080 \\
0.1717 & 0.4586 & 0.2620 & 0.7045 & 0.7552
\end{array}\right]
$$


The beliefs, $\gamma^{h}=\left(\gamma_{1}^{h}, \cdots, \gamma_{S}^{h}\right)$, of the households as to the occurrence of states in date one are given by

$$
\left[\begin{array}{l}
\gamma^{1} \\
\gamma^{2} \\
\gamma^{3} \\
\gamma^{4}
\end{array}\right]=\left[\begin{array}{lllll}
0.1480 & 0.3261 & 0.1375 & 0.2692 & 0.1191 \\
0.1461 & 0.1280 & 0.2999 & 0.2163 & 0.2097 \\
0.2668 & 0.2638 & 0.2651 & 0.1154 & 0.0888 \\
0.2033 & 0.2519 & 0.2892 & 0.1755 & 0.0802
\end{array}\right] .
$$

The collateral requirements of the assets are given by

$$
\left[c^{1}, c^{2}\right]=\left[\begin{array}{ll}
3 & 0 \\
0 & 1 \\
2 & 4
\end{array}\right]
$$

The promises of the assets are given by

$$
A^{1}=\left[\begin{array}{lllll}
1 & 3 & 4 & 2 & 2 \\
1 & 4 & 0 & 3 & 2 \\
4 & 3 & 1 & 2 & 2
\end{array}\right] ; A^{2}=\left[\begin{array}{lllll}
2 & 0 & 3 & 2 & 1 \\
1 & 3 & 2 & 2 & 4 \\
1 & 1 & 0 & 3 & 4
\end{array}\right] ;
$$

where the $s$-th column of $A^{j}$ denotes the promise of asset $j$ in state $s$.

Data of Example 3

The endowments of the households are given by

$$
\begin{aligned}
e^{1} & =\left[\begin{array}{ccccccc}
23 & 1 & 29 & 17 & 48 & 2 & 15 \\
11 & 14 & 25 & 45 & 22 & 50 & 35 \\
49 & 18 & 4 & 25 & 34 & 3 & 35 \\
4 & 46 & 47 & 48 & 27 & 40 & 1 \\
1 & 6 & 23 & 33 & 39 & 32 & 44
\end{array}\right] ; e^{2}=\left[\begin{array}{ccccccc}
32 & 49 & 20 & 43 & 21 & 37 & 15 \\
17 & 18 & 33 & 24 & 45 & 46 & 1 \\
2 & 2 & 49 & 41 & 4 & 10 & 40 \\
18 & 46 & 39 & 44 & 17 & 32 & 10 \\
42 & 11 & 8 & 43 & 14 & 32 & 3
\end{array}\right] ; \\
e^{3} & =\left[\begin{array}{ccccccc}
44 & 3 & 14 & 11 & 30 & 22 & 9 \\
5 & 37 & 5 & 38 & 4 & 21 & 3 \\
19 & 23 & 14 & 9 & 10 & 42 & 30 \\
36 & 48 & 36 & 39 & 7 & 41 & 41 \\
9 & 34 & 21 & 7 & 37 & 41 & 49
\end{array}\right] ; e^{4}=\left[\begin{array}{ccccccc}
24 & 9 & 47 & 7 & 21 & 18 & 27 \\
2 & 19 & 34 & 16 & 26 & 2 & 3 \\
33 & 48 & 50 & 30 & 21 & 34 & 23 \\
9 & 11 & 33 & 31 & 23 & 17 & 23 \\
21 & 19 & 43 & 23 & 20 & 32 & 33
\end{array}\right] ; \\
e^{5} & =\left[\begin{array}{ccccccc}
14 & 28 & 20 & 4 & 4 & 32 & 17 \\
47 & 47 & 12 & 24 & 49 & 49 & 4 \\
47 & 36 & 30 & 13 & 34 & 50 & 46 \\
29 & 34 & 11 & 31 & 7 & 27 & 49 \\
27 & 31 & 12 & 34 & 30 & 37 & 5
\end{array}\right] ; e^{6}=\left[\begin{array}{ccccccc}
47 & 21 & 28 & 17 & 3 & 15 & 12 \\
2 & 14 & 10 & 15 & 30 & 26 & 33 \\
14 & 19 & 21 & 47 & 18 & 41 & 35 \\
14 & 26 & 8 & 45 & 8 & 17 & 25 \\
46 & 14 & 26 & 30 & 41 & 12 & 39
\end{array}\right] ;
\end{aligned}
$$


where the $s$-th column of $e^{h}$ denotes the endowment of household $h$ in state $s-1$. The durability of the commodities are given by

$$
\left[\kappa_{1}, \cdots, \kappa_{6}\right]=\left[\begin{array}{llllll}
0.8394 & 0.0924 & 0.5403 & 0.1291 & 0.1181 & 0.1354 \\
0.6569 & 0.7192 & 0.4090 & 0.4703 & 0.6618 & 0.1897 \\
0.6563 & 0.7367 & 0.6496 & 0.8520 & 0.6441 & 0.9230 \\
0.4099 & 0.4219 & 0.6495 & 0.0433 & 0.8997 & 0.7213 \\
0.3916 & 0.0767 & 0.1358 & 0.2100 & 0.9161 & 0.0749
\end{array}\right] .
$$

The beliefs, $\gamma^{h}=\left(\gamma_{1}^{h}, \cdots, \gamma_{S}^{h}\right)$, of the households as to the occurrence of states in date one are given by

$$
\left[\begin{array}{l}
\gamma^{1} \\
\gamma^{2} \\
\gamma^{3} \\
\gamma^{4} \\
\gamma^{5} \\
\gamma^{6}
\end{array}\right]=\left[\begin{array}{llllll}
0.1943 & 0.2295 & 0.0780 & 0.1434 & 0.2659 & 0.0890 \\
0.2159 & 0.1348 & 0.2563 & 0.0940 & 0.1115 & 0.1876 \\
0.3009 & 0.1479 & 0.1720 & 0.1545 & 0.1433 & 0.0815 \\
0.1754 & 0.1804 & 0.1406 & 0.1025 & 0.2272 & 0.1739 \\
0.1803 & 0.2050 & 0.1056 & 0.1919 & 0.2465 & 0.0707 \\
0.2099 & 0.1258 & 0.1128 & 0.1763 & 0.1694 & 0.2057
\end{array}\right] .
$$

The collateral requirements of the assets are given by

$$
\left[c^{1}, \cdots, c^{5}\right]=\left[\begin{array}{lllll}
3 & 4 & 3 & 0 & 0 \\
1 & 2 & 2 & 2 & 0 \\
1 & 4 & 4 & 1 & 1 \\
4 & 0 & 1 & 4 & 0 \\
1 & 4 & 3 & 0 & 0
\end{array}\right]
$$

The promises of the assets are given by

$$
A^{1}=\left[\begin{array}{llllll}
4 & 4 & 4 & 3 & 0 & 1 \\
4 & 0 & 1 & 3 & 2 & 1 \\
0 & 4 & 1 & 3 & 4 & 4 \\
1 & 3 & 1 & 2 & 1 & 4 \\
0 & 4 & 3 & 0 & 4 & 4
\end{array}\right] ; A^{2}=\left[\begin{array}{llllll}
1 & 2 & 4 & 3 & 3 & 1 \\
2 & 1 & 0 & 0 & 4 & 0 \\
1 & 1 & 3 & 2 & 1 & 2 \\
2 & 3 & 0 & 2 & 3 & 0 \\
0 & 2 & 3 & 4 & 4 & 4
\end{array}\right]
$$




$$
\begin{aligned}
A^{3} & =\left[\begin{array}{llllll}
2 & 2 & 3 & 3 & 2 & 0 \\
1 & 4 & 2 & 3 & 0 & 3 \\
0 & 4 & 2 & 0 & 4 & 1 \\
3 & 4 & 2 & 0 & 2 & 0 \\
0 & 4 & 3 & 2 & 1 & 2
\end{array}\right] ; A^{4}=\left[\begin{array}{llllll}
2 & 1 & 4 & 0 & 0 & 1 \\
0 & 4 & 0 & 4 & 4 & 2 \\
2 & 1 & 4 & 0 & 4 & 3 \\
0 & 2 & 3 & 4 & 1 & 1 \\
3 & 1 & 2 & 4 & 2 & 3
\end{array}\right] \\
A^{5} & =\left[\begin{array}{llllll}
3 & 3 & 4 & 1 & 0 & 4 \\
2 & 2 & 0 & 2 & 4 & 0 \\
3 & 1 & 4 & 4 & 3 & 4 \\
0 & 2 & 0 & 0 & 4 & 4 \\
2 & 0 & 2 & 2 & 4 & 3
\end{array}\right] ;
\end{aligned}
$$

where the $s$-th column of $A^{j}$ denotes the promise of asset $j$ in state $s$.

\section{References}

Allgower, E. and Georg, K. (1994). Numerical Path Following. Colorado State University.

Allgower, E. L. and Georg, K. (1993). Continuation and path following. Acta Numerica, 2:1-64.

Araujo, A., Kuble, r. F., and Schommer, S. (2012). Regulating collateralrequirements when markets are incomplete. Journal of Economic Theory, 147(2):450 - 476 .

Araujo, A., Pascoa, M. R., and Torres-Martinez, J. P. (2002). Collateral avoids ponzi schemes in incomplete markets. Econometrica, 70(4):1613-1638.

Brown, D. J., Demarzo, P. M., and Eaves, B. C. (1996). Computing equilibria when asset markets are incomplete. Econometrica, 64(1):1-27.

Debreu, G. (1959). Theory of Value. Wiley, New York.

Diamond, D. W. (1984). Financial intermediation and delegated monitoring. Review of Economic Studies, 51(3):393-414.

Diamond, P. A. (1967). The role of a stock market in a general equilibrium model with technological uncertainty. The American Economic Review, 57(4):759776.

Dubey, P., Geanakoplos, J., and Shubik, M. (2005). Default and punishment in general equilibrium. Econometrica, 73(1):pp. 1-37. 
Duffie, D. and Shafer, W. (1985). Equilibrium in incomplete markets: I: A basic model of generic existence. Journal of Mathematical Economics, 14(3):285 300 .

Eaves, B. C. (1972). Homotopies for computation of fixed points. Mathematical Programming, 3:1-22.

Eaves, B. C. and Schmedders, K. (1999). General equilibrium models and homotopy methods. Journal of Economic Dynamics and Control, 23(9-10):12491279.

Gale, D. and Hellwig, M. (1985). Incentive-compatible debt contracts: The oneperiod problem. The Review of Economic Studies, 52(4):647-663.

Geanakoplos, J. and Zame, W. (2010). Collateral equilibrium. Cowles Foundation Discussion Paper.

Gottardi, P. and Kubler, F. (2012). Dynamic competitive economies with complete markets and collateral constraints. Economics Working Papers.

Hart, O. D. (1975). On the optimality of equilibrium when the market structure is incomplete. Journal of Economic Theory, 11(3):418-443.

Kellogg, R. B., Li, T. Y., and Yorke, J. (1976). A constructive proof of the brouwer fixed-point theorem and computational results. SIAM Journal on Numerical Analysis, 13(4):473-483.

Magill, M. and Quinzii, M. (1994). Infinite horizon incomplete markets. Econometrica, 62(4):853-80.

Radner, R. (1972). Existence of equilibrium of plans, prices, and price expectations in a sequence of markets. Econometrica, 40(2):289-303.

Sabarwal, T. (2003). Competitive equilibria with incomplete markets and endogenous bankruptcy. Contributions in Theoretical Economics, 3(1):1060-.

Scarf, H. (1967). The approximation of fixed points of a continuous mapping. SIAM Journal on Applied Mathematics, 15(5):1328-1343.

Scarf, H. E. and Hansen, T. (1973). The Computation of Economic Equilibrium. Yale University Press, New Haven.

Smale, S. (1976). A convergent process of price adjustment and global newton methods. Journal of Mathematical Economics, 3(2):107-120. 
Stiglitz, J. E. (1982). The inefficiency of the stock market equilibrium. The Review of Economic Studies, 49(2):241-261.

Townsend, R. M. (1979). Optimal contracts and competitive markets with costly state verification. Journal of Economic Theory, 21(2):265-293.

Wright, M. H. (1992). Interior methods for constrained optimization. Acta Numerica, 1:341-407.

Zame, W. R. (1993). Efficiency and the role of default when security markets are incomplete. The American Economic Review, 83(5):1142-1164.

Zang, I. (1980). A smoothing-out technique for min-max optimization. Mathematical Programming, 19:61-77. 